\title{
Minimal order controllers for output regulation of nonlinear systems
}

\author{
Vivek Natarajan and George Weiss
}

\begin{abstract}
This paper is about the nonlinear local error feedback regulator problem. The plant is a nonlinear finite-dimensional system with a single control input and a single output and it is locally exponentially stable around the origin. The plant is driven, via a separate disturbance input, by a Lyapunov stable exosystem whose states are nonwandering. The reference signal that the plant output must track is a nonlinear function of the exosystem state. The local error feedback regulator problem is to design a dynamic feedback controller, with the tracking error as its input, such that (i) the closed-loop system of the plant and the controller is locally exponentially stable, and (ii) the tracking error tends to zero for all sufficiently small initial conditions of the plant, the controller and the exosystem. Under the assumption that the above regulator problem is solvable, we propose a nonlinear controller whose order is relatively small - typically equal to the order of the exosystem, and which solves the regulator problem. The emphasis is on the low order of the controller. In contrast, previous results on the regulator problem have typically proposed controllers of a much larger order. The stability assumption on the plant (which can be relaxed to some extent) is crucial for making it possible to design a low order controller. We will show, under certain assumptions, that our proposed controller is of minimal order. Three examples are presented - the first illustrates our controller design procedure using an exosystem whose trajectories are periodic even though the state operator of the linearized exosystem contains a nontrivial Jordan block. The second example is more involved, and shows that sometimes a nontrivial immersion of the exosystem is needed in the design. The third example, based on output voltage regulation for a boost power converter, shows how the regulator equations may reduce to a first order PDE with no given boundary conditions, but which nevertheless has a locally unique solution.
\end{abstract}

Keywords. Lyapunov stable nonlinear exosystem, nonwandering points, error feedback regulator problem, center manifold, regulator equations, minimal order controller, internal model, nontrivial Jordan block, boost converter, quasilinear PDE.

\section{Introduction}

Given a stabilizable plant and an unstable exosystem, the local error feedback regulator problem is to design a locally stabilizing controller (for the plant) which guarantees the tracking of certain reference signals by the plant output, even when the plant is driven by external disturbance signals. The reference and disturbance

This work was partially supported by grant no. 800/14 of the Israel Science Foundation.

V. Natarajan (vivek.natarajan@iitb.ac.in) is with the Systems and Control Engineering Group, Indian Institute of Technology Bombay, Mumbai, India, 400076, Ph:+91 2225765385

G. Weiss (gweiss@eng.tau.ac.il) is with the School of Electrical Engineering, Tel Aviv University, Ramat Aviv, Israel, 69978, Ph:+972 36405164. 
signals are both functions of the exosystem state, assumed to be sufficiently small. The input to the controller is the tracking error and its output is the control input to the plant. The robust version of the regulator problem is to design a controller that guarantees both stabilization and tracking for a set of uncertain plants.

The regulator problem, and its robust version, for linear finite-dimensional plants and exosystems was addressed in [16] and [15] using geometric methods. The solvability of the problem was characterized in terms of the solvability of certain matrix equations, known in the literature as the regulator equations. The internal model principle, which states that for robust regulation the dynamic structure of the exosystem (suitably duplicated) must be incorporated into the controller, was introduced in [16]. Following these works, the regulator problem for nonlinear finitedimensional plants and exosystems was addressed in [24] in a local setting, i.e. the proposed controller ensured that its closed-loop system with the plant is locally exponentially stable and that tracking is achieved for sufficiently small initial conditions of the plant, the controller and the exosystem. Nonlinear regulator equations, a generalization of the regulator equations in [15], were introduced in [24].

Since the early 1990's many researchers have extended the results in [24], mainly by developing controllers that solve the nonlinear robust regulator problem in local $([39,40,8])$, semi-global $([29$, 23, 46]) and global ([45, 11, 49]) settings. Most of these works (excluding [29]) address the robust regulator problem using the regulator equations. The semi-global and global results mentioned above are obtained for plants that are single-input single-output (SISO) from control input to output; the recent works [38, 25, 3] consider nonlinear multi-input multi-output plants. All the aforementioned nonlocal results are derived for plants that possess additional properties (such as minimum-phase, feedback linearizable, etc). Another global result is in [27], where a rather restrictive regulator problem was solved assuming that the plant is passive. Related results for fully actuated mechanical systems with unknown parameters are in [26]. Using ideas from the nonlinear regulator theory, a novel method for the stabilization and adaptive control of nonlinear systems, called immersion and invariance, was developed in [4]. For a comprehensive introduction to the nonlinear regulator problem, see [7, 21, 22].

In this paper we focus on finding a minimal order (or at least, low order) controller that solves the nonlinear error feedback regulator problem in a local setting. Specifically, assuming a locally stable nonlinear plant that is SISO from control input to output (but it may also have disturbance inputs) and a Lyapunov stable nonlinear exosystem whose states are nonwandering, connected as shown in Figure 2, we will construct controllers whose order is the same as that of a certain immersion of the exosystem. In many applications, this immersion is just a copy of the exosystem. The order of the controllers in the papers mentioned earlier, even in the absence of uncertain plant parameters, is typically equal to or larger than that of the exosystem and the plant combined. A key reason for this, apart from the fact that the control objectives in those papers are different, is the sequential control design approach they adopt. In their approach, first an internal model (whose order can be larger than that of the exosystem) is designed and then the loop containing the internal 
model and the plant is stabilized using an additional controller. In contrast we will design a stabilizing internal model directly. The search for minimal order controllers is of practical value from an implementation standpoint, and is also of theoretical interest. For a discussion on the lower bound for the order of any controller that solves the linear robust regulator problem see [13, 14].

Our work is motivated by an alternate approach to the linear regulator problem, first proposed in 12] for stable finite-dimensional linear plants. In this approach, the control and observation operators of the internal model (whose order is $r \times m$, where $r$ is the number of outputs and $m$ is the exosystem order) are chosen to ensure that its closed-loop system with the plant is stable. Hence no additional stabilizing controller is required and this may lead to the construction of minimal order robust controllers for the linear regulator problem.. This approach was extended in [19, 41] to construct finite-dimensional controllers that solve the regulator problem for stable linear infinite-dimensional plants. We will extend this approach to nonlinear finitedimensional plants that are SISO from control input to output. Preliminary versions of this work have appeared in [37, 48], see Remark 2.2.

In Section 2 we introduce the nonlinear error feedback regulator problem and present relevant background information. This includes Definitions 2.6 and 2.7]which describe the notions of autonomous systems and their local immersions. We then state Theorem 2.8, a well-known result about the connection between the solvability of the local error feedback regulator problem and the local solvability of the regulator equations. This theorem is somewhat more general and precise than other versions in the literature, see Remark 2.9. Finally we state Proposition 2.10 about the linear error feedback regulator problem, which follows from the results in [19].

In Section 3, which is for technical preparation, we state a version of the classical center manifold theorem. Using this theorem we prove Proposition 3.2 which shows that for certain autonomous systems, given a local immersion, there exists a possibly lower order local immersion with special spectral properties.

Section 4 contains our main result, Theorem 4.1, which states the existence of a low order controller under some natural assumptions. This controller contains a suitable local immersion of an autonomous system constructed using the exosystem and a solution of the regulator equations. The order of the controller is equal to the order of the immersion. The input map of the controller is linear. Under some detectability assumptions on the linearized plant and linearized exosystem, this controller is of minimal order, see Corollary 4.3.

Section 5 contains three examples. In the first example we present a nonlinear exosystem, the state operator of whose linearization at the origin contains a non-trivial Jordan block. We show that this exosystem is Lyapunov stable and all its trajectories are periodic. We then illustrate our controller design using this exosystem and a second-order plant. The second example shows that in some cases there must exist a local immersion for the exosystem, with order larger than that of the exosystem, for the regulator problem to have a solution. We then present an immersion such that the controller constructed using it is of minimal order. The third example is output 
voltage regulation for the boost power converter shown in Figure 1. A constant but unknown input voltage $v>0$ is transformed into a higher voltage $z_{1}$ that feeds a load $R$. Due to the fast switching, there will be high frequency ripple on $z_{1}$, which becomes negligible for very high switching frequency, and we neglect this ripple. The control problem is to make $z_{1}$ track a reference value, in spite of the sinusoidal disturbance current $i_{e}$. After putting this system into our framework, we shall see that the regulator equations reduce to a first order PDE with no given boundary conditions, but which nevertheless has a locally unique solution.

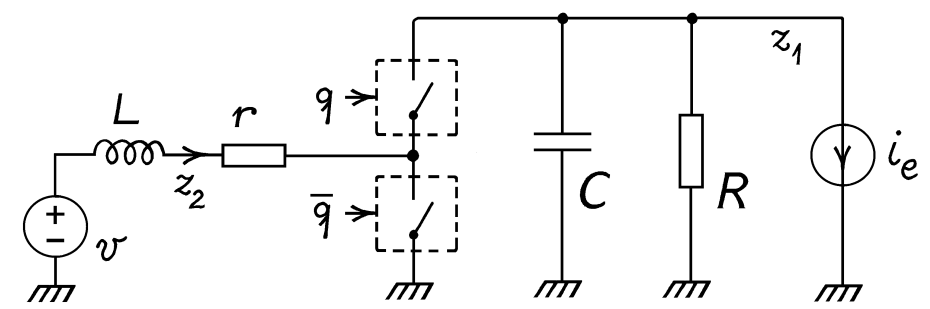

Figure 1. A boost converter with input voltage $v$, input current $z_{2}$, output voltage $y=z_{1}$ and disturbance current $i_{e}$. The electronic switches are controlled by the binary signals $q$ and $\bar{q}=1-q$, generated by a controller.

\section{Problem setting and background}

In this section, following [22], we introduce the nonlinear plant and exosystem in Subsection 2.1 and define the error feedback regulator problem for them in Subsection 2.2. We present the definition of autonomous systems and of their local immersions in Subsection 2.3 and then restate a well-known result from [22] which characterizes the solvability of the regulator problem, but with added details about the smoothness of certain maps. Finally, considering a linearization of the plant and the exosystem, we present a simple proposition in Subsection 2.4 which follows immediately from the results in [12, 19].

2.1 The plant and the exosystem. We consider a nonlinear plant

$$
\dot{x}=f(x, u, w), \quad y=g(x, u, w),
$$

with state $x(t) \in X \subset \mathbb{R}^{n}$, control input $u(t) \in U \subset \mathbb{R}$ and output $y(t) \in Y \subset \mathbb{R}$, where $X, U$ and $Y$ are open sets that contain the origin of the appropriate spaces. The exogenous disturbance signal $w$ in (2.1) is the state of the nonlinear exosystem

$$
\dot{w}=s(w),
$$

with $w(t) \in W \subset \mathbb{R}^{p}$, where $W$ is an open set containing the origin. The functions $f: X \times U \times W \rightarrow \mathbb{R}^{n}, g: X \times U \times W \rightarrow Y$ and $s: W \rightarrow W$ are assumed to be of class $C^{2}$ with $f(0,0,0)=0, g(0,0,0)=0$ and $s(0)=0$. The reference signal is

$$
y_{r}=q(w) .
$$

The function $q: W \rightarrow Y$ is assumed to be of class $C^{2}$ with $q(0)=0$. The tracking error is

$$
e(t)=y(t)-y_{r}(t)=g(x, u, w)-q(w)=h(x, u, w) .
$$


Clearly $h: X \times U \times W \rightarrow Y_{e}$ is a $C^{2}$ map satisfying $h(0,0,0)=0$. Here $Y_{e} \subset \mathbb{R}$ is open and $Y \subset Y_{e}$. While we consider only SISO plants, the controller design procedure described in this work also applies to MIMO plants. However, it is unclear if the resulting controller will be of minimal order. This is a topic for future research.

For the dynamics of the exosystem, we assume that the origin is a Lyapunov stable equilibrium point and also that every point $w_{0} \in W$ is nonwandering. If $\phi$ denotes the flow generated by $s$ on $W$, then a point $w_{0} \in W$ is called nonwandering if its trajectory $\phi_{t}\left(w_{0}\right)$ is defined for all $t>0$ and for every neighborhood $\mathcal{O}$ of $w_{0}$ and every $T>0$, there exists $\tau>T$ such that $\phi_{\tau}(\mathcal{O}) \cap \mathcal{O}$ is not empty (see [18, Definition 5.2.2, page 236]). Equivalently, for each $w_{0} \in W$ there exist sequences $\left(t_{n}\right)_{n=1}^{\infty}$ in $(0, \infty)$ and $\left(w_{n}\right)_{n=1}^{\infty}$ in $W$ such that $\phi_{t_{n}}\left(w_{n}\right)$ exists and

$$
t_{n} \rightarrow \infty, \quad w_{n} \rightarrow w_{0} \quad \text { and } \quad \phi_{t_{n}}\left(w_{n}\right) \rightarrow w_{0} .
$$

Denoting $\tilde{t}_{n}=-t_{n}, \tilde{w}_{n}=\phi_{t_{n}}\left(w_{n}\right)$, it follows that $\phi_{\tilde{t}_{n}}\left(\tilde{w}_{n}\right)=w_{n}$ exists and

$$
\tilde{t}_{n} \rightarrow-\infty, \quad \tilde{w}_{n} \rightarrow w_{0} \quad \text { and } \quad \phi_{\tilde{t}_{n}}\left(\tilde{w}_{n}\right) \rightarrow w_{0},
$$

which is exactly the same property as (2.5) but backwards in time. We mention that [22, 24] make a slightly stronger assumption about the exosystem: every point $w_{0} \in W$ is assumed to be Poisson stable, which is like (2.5) with $w_{n}=w_{0}$, together with (2.6) with $\tilde{w}_{n}=w_{0}$.

We define the real matrices

$$
\begin{gathered}
A=\left[\frac{\partial f}{\partial x}\right]_{(0,0,0)}, \quad B=\left[\frac{\partial f}{\partial u}\right]_{(0,0,0)}, \quad P=\left[\frac{\partial f}{\partial w}\right]_{(0,0,0)}, \\
C=\left[\frac{\partial h}{\partial x}\right]_{(0,0,0)}, \quad D=\left[\frac{\partial h}{\partial u}\right]_{(0,0,0)}, \quad Q=\left[\frac{\partial h}{\partial w}\right]_{(0,0,0)}, \quad S=\left[\frac{\partial s}{\partial w}\right]_{(0)} .
\end{gathered}
$$

The assumptions on $s$ imply that $S$ has all its eigenvalues on the imaginary axis (see Remark 8.1.1 in 22] for a closely related statement, with practically the same proof). If $s$ is linear, then the assumptions on $s$ also imply that $S$, when written in the Jordan normal form, has no Jordan blocks of order larger than 1. However, for a nonlinear $s, S$ can have nontrivial Jordan blocks; for instance, see the exosystem in Example 5.1. Our main result is stated under the following assumption, which can be relaxed (this is discussed in Remark 4.5). For any square matrix $M$, let $\sigma_{p}(M)$ denote the set of eigenvalues of $M$ and let $\rho(M)=\left\{a \in \mathbb{C} \mid a \notin \sigma_{p}(M)\right\}$.

Assumption 2.1. The matrix $A$ is stable (i.e. $\operatorname{Re} \lambda<0$ for all $\lambda \in \sigma_{p}(A)$ ).

The above assumption is natural given the fact that this work, as discussed in the Introduction, builds on the results in [12, 19, 41] (see Proposition 2.10) which have been developed for exponentially stable plants. We will need the following linearization at $(x, u, w)=(0,0,0)$ of the plant (2.1) and the error (2.4):

$$
\begin{aligned}
\dot{x} & =A x+B u+P w, \\
e_{l} & =C x+D u+Q w .
\end{aligned}
$$

The transfer function of the linear system (2.7), (2.8) from the control input $u$ to the linearized error $e_{l}$ is $\mathbf{G}(z)=C(z I-A)^{-1} B+D$, which is defined for each $z \in \rho(A)$. 
Remark 2.2. This work generalizes the results in [37, 48] by relaxing the assumption in those papers that the pair $\left(\left[\begin{array}{ll}C & Q\end{array}\right],\left[\begin{array}{ll}A & P \\ 0 & S\end{array}\right]\right)$ be detectable. This higher generality makes the main result and its proof much more complicated. In particular, we have to consider local immersions of autonomous systems (see Definitions 2.6 and 2.7) and derive new results for them using the center manifold theorem (see Proposition 3.2). Furthermore, the exosystem in [37, 48] was linear with only simple eigenvalues, but these conditions are dropped in the present work, at the expense of new, more complex arguments. Letting $S$ to contain non-trivial Jordan blocks allowed us to explore an interesting and, to the best of our knowledge, novel structure for the exosystem in Example [5.1. Finally, two other examples from [37, 48] have been further refined and expanded in this work.

2.2 The error feedback regulator problem. Consider the controller

$$
\dot{\xi}=\eta(\xi, e), \quad u=\theta(\xi),
$$

with state $\xi(t) \in X_{c} \subset \mathbb{R}^{n_{c}}$, input $e$ and output $u$, where $X_{c}$ is open and contains 0 . The functions $\eta: X_{c} \times Y_{e} \rightarrow \mathbb{R}^{n_{c}}$ and $\theta: X_{c} \rightarrow U$ are $C^{2}$ maps satisfying $\eta(0,0)=0$ and $\theta(0)=0$. The closed-loop system consisting of the plant (2.1), the exosystem (2.2) and the controller (2.9) is shown in Figure 2. We define the matrices

$$
F=\left[\frac{\partial \eta}{\partial \xi}\right]_{(0,0)}, \quad G=\left[\frac{\partial \eta}{\partial e}\right]_{(0,0)}, \quad K=\left[\frac{\partial \theta}{\partial \xi}\right]_{(0)},
$$

which determine the linearization of the controller. The order of this controller is, by definition, $n_{c}$.

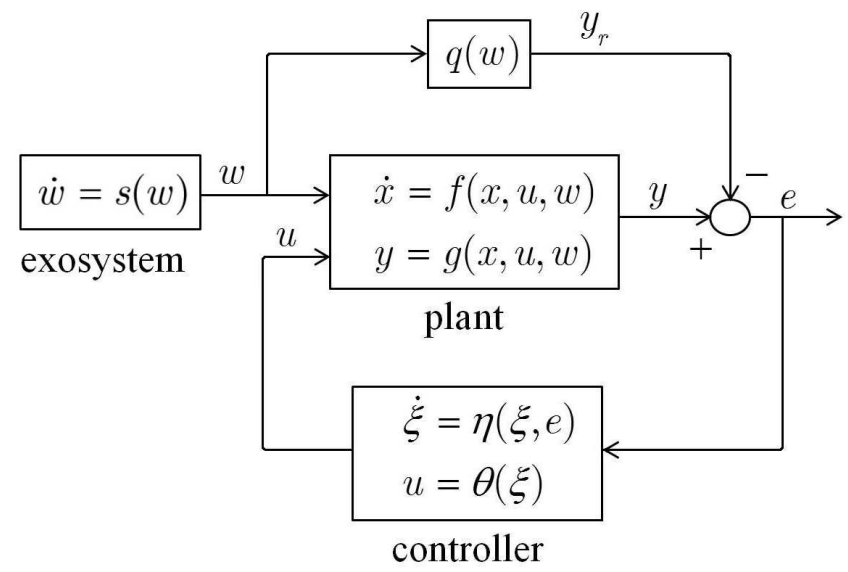

Figure 2. The closed-loop system of the plant and the controller, driven by the exosystem

Definition 2.3. The controller (2.9) is said to solve the local error feedback regulator problem for the plant (2.1), the exosystem (2.2) and the error (2.4) if:

1. The equilibrium $(x, \xi)=(0,0)$ of the unforced closed-loop system

$$
\dot{x}=f(x, \theta(\xi), 0), \quad \dot{\xi}=\eta(\xi, h(x, \theta(\xi), 0)),
$$

is locally exponentially stable. 
2. The forced closed-loop system

$$
\dot{x}=f(x, \theta(\xi), w), \quad \dot{w}=s(w), \quad \dot{\xi}=\eta(\xi, h(x, \theta(\xi), w)),
$$

is such that for each initial condition $(x(0), \xi(0), w(0))$ in some fixed neighborhood of $(0,0,0)$ in $X \times X_{c} \times W$, it has a unique global (in time $t \geq 0$ ) solution $(x, \xi, w)$ and the corresponding error $e$ defined in (2.4) satisfies

$$
\lim _{t \rightarrow+\infty} e(t)=\lim _{t \rightarrow+\infty} h(x(t), \theta(\xi(t)), w(t))=0 .
$$

2.3 The regulator equations. Lemma 2.4 and Theorem 2.8 , presented below, are well-known results in the nonlinear regulator theory. They were first established in 24] under certain detectability assumptions on the linearization of the plant, the error and the exosystem. A more general version of Theorem 2.8 (along with Definition 2.7) was then presented in 22]. Lemma 2.4 and Theorem 2.8 do not require that $A$ be stable. Also, these results are derived in [22] for multi-input multi-output plants. For some comments on these results, see Remark 2.9.

Lemma 2.4. Suppose that for the controller (2.9) the first condition in Definition 2.3 (local exponential stability) holds. This controller solves the local error feedback regulator problem for the plant (2.1), the exosystem (2.2) and the error (2.4) if and only if there exist an open set $W^{o} \subset W$ containing zero and $C^{2}$ maps $\pi: W^{o} \rightarrow X$ and $\sigma: W^{o} \rightarrow X_{c}$, with $\pi(0)=0$ and $\sigma(0)=0$, satisfying for every $w \in W^{o}$

$$
\begin{aligned}
& \frac{\partial \pi}{\partial w} s(w)=f(\pi(w), \theta(\sigma(w)), w), \\
& \frac{\partial \sigma}{\partial w} s(w)=\eta(\sigma(w), 0), \\
& h(\pi(w), \theta(\sigma(w)), w)=0 .
\end{aligned}
$$

Moreover, in this case there exist open sets $Z \subset X \times X_{c}$ and $W^{o o} \subset W^{o}$, both containing zero, such that for any $\left(x_{0}, \xi_{0}\right) \in Z$ and any $w_{0} \in W^{o o}$, the closed-loop system (2.11) has a unique global (in time $t \geq 0$ ) solution $(x, \xi, w)$ with $x(0)=x_{0}$, $\xi(0)=\xi_{0}, w(0)=w_{0}$ and

$$
\lim _{t \rightarrow \infty}\|x(t)-\pi(w(t))\|=0, \quad \lim _{t \rightarrow \infty}\|\xi(t)-\sigma(w(t))\|=0 .
$$

Remark 2.5. The statement of the above lemma in [22] does not describe the asymptotic response of the plant and the controller states expressed by the limits in (2.15). These limits can be obtained using the center manifold theory [10] which guarantees the existence of a locally attractive invariant manifold $\{(\pi(w), \sigma(w), w) \mid$ $\left.w \in W^{o o}\right\} \subset Z \times W^{o o}$ for the dynamics of the closed-loop system (2.11).

Definition 2.6. An autonomous system is a triple $\left(W_{1}, s_{1}, \gamma_{1}\right)$, where $W_{1} \subset \mathbb{R}^{r_{1}}$ is an open set containing the origin and the maps $s_{1}: W_{1} \rightarrow \mathbb{R}^{r_{1}}$ and $\gamma_{1}: W_{1} \rightarrow \mathbb{R}$ are both of class $C^{2}$ and they satisfy $s_{1}(0)=0$ and $\gamma_{1}(0)=0$. The order of this system is $r_{1}$. The state trajectories $w_{1}$ and the corresponding output functions $u_{1}$ of $\left(W_{1}, s_{1}, \gamma_{1}\right)$ are the solutions (on any time interval) of the equations

$$
\dot{w}_{1}=s_{1}\left(w_{1}\right), \quad u_{1}=\gamma_{1}\left(w_{1}\right)
$$

with $w_{1}(t) \in W_{1}$ and $u_{1}(t) \in \mathbb{R}$. 
Note that the triple $(W, s, q)$ from (2.2) and (2.3) is an autonomous system.

Definition 2.7. An autonomous system $\left(W_{1}, s_{1}, \gamma_{1}\right)$ is said to be locally immersed into another autonomous system $\left(W_{2}, s_{2}, \gamma_{2}\right)$ if there exists an open set $\widetilde{W}_{1} \subset W_{1}$ containing the origin and a $C^{2}$ map $\tau: \widetilde{W}_{1} \rightarrow W_{2}$ such that $\tau(0)=0$ and

$$
\frac{\partial \tau}{\partial w_{1}} s_{1}\left(w_{1}\right)=s_{2}\left(\tau\left(w_{1}\right)\right), \quad \gamma_{1}\left(w_{1}\right)=\gamma_{2}\left(\tau\left(w_{1}\right)\right) \quad \forall w_{1} \in \widetilde{W}_{1} .
$$

For systems as above, all the output functions of $\left(\widetilde{W}_{1}, s_{1}, \gamma_{1}\right)$ are also output functions of $\left(W_{2}, s_{2}, \gamma_{2}\right)$. However, the eigenvalues of the linearization of $s_{1}$ need not be eigenvalues of the linearization of $s_{2}$, see Example 5.2 .

Theorem 2.8. (Isidori and Byrnes) There exists a controller of the form (2.9) that solves the local error feedback regulator problem for the plant (2.1), the exosystem (2.2) and the error (2.4) if and only if there exist an open set $W^{\circ} \subset W$ with $0 \in W^{o}$ and $C^{2}$ maps $\pi: W^{o} \rightarrow X$ and $\gamma: W^{o} \rightarrow U$, with $\pi(0)=0$ and $\gamma(0)=0$, such that:

1. For each $w \in W^{o}, \pi$ and $\gamma$ satisfy the nonlinear regulator equations

$$
\begin{aligned}
& \frac{\partial \pi}{\partial w} s(w)=f(\pi(w), \gamma(w), w), \\
& h(\pi(w), \gamma(w), w)=0 .
\end{aligned}
$$

2. The autonomous system $\left(W^{o}, s, \gamma\right)$ is locally immersed into $\left(E^{o}, \phi, \lambda\right)$, and the linearization of the latter system, $\Phi=\left[\frac{\partial \phi}{\partial \zeta}\right]_{(0)}$ and $\Lambda=\left[\frac{\partial \lambda}{\partial \zeta}\right]_{(0)}$, is such that for some column vector $N$ (of suitable dimension),

$$
\left(\left[\begin{array}{cc}
A & B \Lambda \\
N C & \Phi+N D \Lambda
\end{array}\right],\left[\begin{array}{c}
B \\
0
\end{array}\right],\left[\begin{array}{cc}
C & D \Lambda
\end{array}\right]\right) \text { is stabilizable and detectable. }
$$

The intuitive meaning of the system (2.19) can be understood from Proposition 2.10 below: it is the linearization of the feedback interconnection of the plant (2.1) with the internal model $\left(E^{o}, \phi, \lambda\right)$, the latter receiving its input $e$ via the matrix $N$.

Suppose that the conditions in Theorem 2.8 hold. Let the matrices $K, L$ and $M$ be such that

$$
\left[\begin{array}{ccc}
A & B \Lambda & B M \\
N C & \Phi+N D \Lambda & 0 \\
L C & L D \Lambda & K
\end{array}\right]
$$

is exponentially stable. Then the controller (2.9) with

$$
\xi=\left[\begin{array}{ll}
\xi_{0} & \xi_{1}
\end{array}\right]^{\top}, \quad \eta(\xi, e)=\left[\begin{array}{c}
K \xi_{0}+L e \\
\phi\left(\xi_{1}\right)+N e
\end{array}\right], \quad \theta(\xi)=M \xi_{0}+\lambda\left(\xi_{1}\right),
$$

solves the local error feedback regulator problem for the plant (2.1), the exosystem (2.2) and the error (2.4) [22. A possible choice for $K, L$ and $M$, suggested by the observer-based controller design approach, is to choose $L$ and $M$ such that

$$
\left[\begin{array}{cc}
A & B \Lambda \\
N C & \Phi+N D \Lambda
\end{array}\right]+\left[\begin{array}{c}
B \\
0
\end{array}\right] M \quad \text { and } \quad\left[\begin{array}{cc}
A & B \Lambda \\
N C & \Phi+N D \Lambda
\end{array}\right]-L\left[\begin{array}{ll}
C & D \Lambda
\end{array}\right]
$$


are exponentially stable and then choose

$$
K=\left[\begin{array}{cc}
A & B \Lambda \\
N C & \Phi+N D \Lambda
\end{array}\right]+\left[\begin{array}{c}
B \\
0
\end{array}\right] M-L\left[\begin{array}{ll}
C & D \Lambda
\end{array}\right] .
$$

In this design, the order of the controller (the dimension of $\xi$ ) is $n_{c}=n+2 \nu$, where $\nu$ is the order of $\left(E^{o}, \phi, \lambda\right)$.

Remark 2.9. The function $g$ in (2.1) is considered in [22] to be independent of $u$ and thus $D=0$ in that work, which makes the formulas simpler. However, the proof remains almost the same if we include the matrix $D$. In [22] there is a different assessment of the smoothness of $\pi$ and $\gamma\left(C^{1}\right.$ in place of $\left.C^{2}\right)$. The $C^{2}$ smoothness can be established by a similar argument.

2.4 Solution to the linear regulator problem. The following proposition is based on the results in [12] which address the linear error feedback regulator problem. Theorem 6 in [19] (and also [41]) extends the results in [12] to a class of infinite-dimensional systems. To simplify our presentation, we state this proposition under the assumption that $0 \in \sigma_{p}(S)$, but the proposition remains true even when this assumption is dropped. Recall the notation $\mathbf{G}$ introduced after (2.8).

Proposition 2.10. Suppose that $A$ is stable and $S$ has eigenvalues $\left\{\alpha_{0}, \pm i \alpha_{1}, \ldots \pm\right.$ $\left.i \alpha_{q}\right\}$, with $\alpha_{0}=0$ and each $\alpha_{j} \in \mathbb{R}$, such that the geometric multiplicity of every eigenvalue is 1 and $\mathbf{G}\left(i \alpha_{j}\right) \neq 0$ for all $j \in\{0,1, \ldots q\}$. Let the vector $C_{c}^{\top} \in \mathbb{R}^{p}$ be such that the pair $\left(C_{c}, S\right)$ is detectable (equivalently, observable). Then there exists a vector $B_{c} \in \mathbb{R}^{p}$ such that the linear controller

$$
\dot{z}_{c}=S z_{c}+B_{c} e_{l}, \quad u=C_{c} z_{c},
$$

for the linearized plant (2.7), (2.8) renders the closed-loop system stable, i.e.

$$
\mathcal{A}=\left[\begin{array}{cc}
A & B C_{c} \\
B_{c} C & S+B_{c} D C_{c}
\end{array}\right]
$$

is a stable matrix. For any such $B_{c}, C_{c}$, the controller in (2.20) solves the linear error feedback regulator problem for the linearized plant (2.7), the linearization of the exosystem (2.2) and the linearized error (2.8), and moreover in this case the linearized error $e_{l}(t)$ converges to zero exponentially.

On the basis of the results in [19], the next remark states a concrete way of choosing $B_{c}$ in the above proposition so that $\mathcal{A}$ in (2.21) is stable.

Remark 2.11. For each $j \in\{0,1, \ldots q\}$, denote by $m_{j}$ the algebraic multiplicity of $i \alpha_{j} \in \sigma_{p}(S)$. Then clearly $p=m_{0}+2 \sum_{j=1}^{q} m_{j}$. For each $j \in\{0,1, \ldots q\}$, choose the set $\left\{a_{j 1}, a_{j 2}, \ldots a_{j m_{j}}\right\} \subset \mathbb{C}$ such that all the zeros of the polynomial $z^{m_{j}}+\mathbf{G}\left(i \alpha_{j}\right) \sum_{k=1}^{m_{j}} a_{j k} z^{m_{j}-k}$ lie in the open left half-plane. Since $\mathbf{G}(0) \in \mathbb{R}$, we can choose $\left\{a_{01}, a_{02}, \ldots a_{0 m_{0}}\right\} \subset \mathbb{R}$. According to [19, Theorem 6], if we choose $B_{c}$ such that the transfer function $\mathbf{C}$ of the linear controller (2.20) is given by 


$$
-\mathbf{C}(z)=\sum_{j=0}^{q} \sum_{k=1}^{m_{j}} \frac{a_{j k} \varepsilon^{k}}{\left(z-i \alpha_{j}\right)^{k}}+\sum_{j=1}^{q} \sum_{k=1}^{m_{j}} \frac{\bar{a}_{j k} \varepsilon^{k}}{\left(z+i \alpha_{j}\right)^{k}},
$$

where $\varepsilon>0$ is a constant and a bar indicates a complex conjugate, then for all $\varepsilon$ sufficiently small $\mathcal{A}$ in (2.21) is stable.

Given $S$ and $C_{c}$, we now describe how $B_{c}$ can be chosen so that the transfer function of (2.20) is C. Let $J$ be an upper-triangular Jordan normal form for $S$, with the set of Jordan blocks on its diagonal being $\left\{J_{0}, J_{1}, J_{-1}, \ldots J_{q}, J_{-q}\right\}$ (in this order, $J_{j}$ and $J_{-j}$ correspond to the eigenvalues $i \alpha_{j}$ and $-i \alpha_{j}$, respectively). Let

$$
T=\left[\begin{array}{llllll}
V_{0} & V_{1} & V_{-1} & \ldots & V_{q} & V_{-q}
\end{array}\right] \in \mathbb{C}^{p \times p}
$$

be the matrix of generalized eigenvectors of $S$ such that $J=T^{-1} S T, V_{0} \in \mathbb{R}^{p \times m_{0}}$ and $V_{-j} \in \mathbb{C}^{p \times m_{j}}$ is the element-wise complex conjugate of $V_{j} \in \mathbb{C}^{p \times m_{j}}$ for all $j \in\{1,2, \ldots q\}$. Define $\mathcal{C}_{c}=C_{c} T$. Then

$$
\mathcal{C}_{c}=\left[\begin{array}{lllll}
\mathcal{C}_{0} & \mathcal{C}_{1} & \mathcal{C}_{-1} & \ldots \mathcal{C}_{q} & \mathcal{C}_{-q}
\end{array}\right] \in \mathbb{C}^{1 \times p}
$$

where $\mathcal{C}_{0} \in \mathbb{R}^{1 \times m_{0}}$ and $\mathcal{C}_{j} \in \mathbb{C}^{1 \times m_{j}}$ and $\mathcal{C}_{-j}$ is the element-wise complex conjugate of $\mathcal{C}_{j}$ for each $j \in\{1,2, \ldots q\}$. We will construct $\mathcal{B}_{c} \in \mathbb{C}^{p \times 1}$ with the structure

$$
\mathcal{B}_{c}^{\top}=\left[\begin{array}{llll}
\mathcal{B}_{0}^{\top} & \mathcal{B}_{1}^{\top} & \mathcal{B}_{-1}^{\top} \ldots \mathcal{B}_{q}^{\top} \mathcal{B}_{-q}^{\top}
\end{array}\right]
$$

where $\mathcal{B}_{0} \in \mathbb{R}^{m_{0} \times 1}$ and $\mathcal{B}_{j} \in \mathbb{C}^{m_{j} \times 1}$ and $\mathcal{B}_{-j}$ is the element-wise complex conjugate of $\mathcal{B}_{j}$ for each $j \in\{1,2, \ldots q\}$, such that

$$
\mathcal{C}_{j}\left(z I-J_{j}\right)^{-1} \mathcal{B}_{j}=\sum_{k=1}^{m_{j}} \frac{-a_{j k} \varepsilon^{k}}{\left(z-i \alpha_{j}\right)^{k}} \quad \forall j \in\{0,1, \ldots q\} .
$$

Using (2.22) and (2.23) it follows that $\mathcal{C}_{c}(z I-J)^{-1} \mathcal{B}_{c}=\mathbf{C}(z)$ for all $z \notin \sigma_{p}(S)$. If we let $B_{c}=T \mathcal{B}_{c}$, it is then easy to verify that $B_{c} \in \mathbb{R}^{p \times 1}$ and $C_{c}(z I-S)^{-1} B_{c}=\mathbf{C}(z)$ for all $z \notin \sigma_{p}(S)$, i.e. for this choice of $B_{c}$ the matrix $\mathcal{A}$ is stable. It now only remains to choose $\mathcal{B}_{j}$ such that (2.23) holds. This is done below.

Fix $j \in\{0,1, \ldots q\}$ and $\varepsilon>0$ sufficiently small. Let $\mathcal{B}_{j}^{\top}=\left[\begin{array}{llll}b_{j 1} & b_{j 2} & \ldots & b_{j m_{j}}\end{array}\right]$ and $\mathcal{C}_{j}=\left[\begin{array}{llll}c_{j 1} & c_{j 2} & \ldots & c_{j m_{j}}\end{array}\right]$. Solve the following algebraic equations for $\left\{b_{j 1}, b_{j 2}, \ldots b_{j m_{j}}\right\}$ :

$$
\sum_{l=1}^{m_{j}-k+1} c_{j l} b_{j(l+k-1)}=-a_{j k} \varepsilon^{k} \quad \forall k \in\left\{1,2, \ldots m_{j}\right\} .
$$

This set of equations has a unique solution. Indeed, since $\left(C_{c}, S\right)$ is a detectable pair, so is $\left(\mathcal{C}_{c}, J\right)$. Hence $\left(\mathcal{C}_{j}, J_{j}\right)$ is also a detectable pair, which implies that $c_{j 1} \neq 0$. The solution to (2.24) can be computed sequentially as follows: $b_{j m_{j}}=-\varepsilon^{m_{j}} a_{j m_{j}} / c_{j 1}$ and given $\left\{b_{j(k+1)}, b_{j(k+2)}, \ldots b_{j m_{j}}\right\}$ for any $1 \leq k \leq m_{j}-1$,

$$
b_{j k}=-\left(a_{j k} \varepsilon^{k}+\sum_{l=2}^{m_{j}-k+1} c_{j l} b_{j(l+k-1)}\right) / c_{j 1} .
$$

It is straightforward to verify that for $\mathcal{B}_{j}$ chosen such that (2.24) holds, (2.23) holds. 
Remark 2.12. Let us consider for the moment the frequently encountered situation when $m_{j}=1$ for all $j \in\{0,1, \ldots q\}$ (i.e., $S$ can be diagonalized). Then the method described in Remark 2.11 becomes much simpler. We write $a_{j}$ instead of $a_{j 1}$. The numbers $a_{j}$ must be chosen such that $\operatorname{Re}\left[a_{j} \mathbf{G}\left(i \alpha_{j}\right)\right]>0$. The transfer function $\mathbf{C}$ of the linear controller from (2.20) should be given by

$$
-\mathbf{C}(z)=\frac{\varepsilon a_{0}}{z}+\varepsilon \sum_{j=1}^{q}\left(\frac{a_{j}}{z-i \alpha_{j}}+\frac{\bar{a}_{j}}{z+i \alpha_{j}}\right),
$$

with $\varepsilon>0$ sufficiently small, and the vector $B_{c}$ must be chosen accordingly. Under a passivity assumption on the plant, any $\varepsilon>0$ will work, see [41].

Remark 2.13. The transfer function of the controller in (2.20) has poles at $\pm i \alpha_{j}$ $(j \in\{0,1, \ldots p\})$. The necessity of the condition $\mathbf{G}\left(i \alpha_{j}\right) \neq 0$ for the solvability of the local error feedback regulator problem can be seen also from the following fact: in a stable closed-loop system there cannot be an unstable pole-zero cancelation in the product of the plant and controller transfer functions. This is well-known but not easy to find in the literature, see for instance [2].

\section{Reduced order local immersion}

We start this section by stating (in Theorem 3.1) a version of the center manifold theorem based on [20, Theorem 2.9 and Corollary 2.12]. We remark that several hypothesis in [20], which considers infinite-dimensional plants, hold trivially for finite-dimensional plants (see [20, Exercise 2.8]). Afterwards we show (in Proposition 3.2) that for certain autonomous systems $\left(W_{1}, s_{1}, \gamma_{1}\right)$ a given local immersion $\left(W_{2}, s_{2}, \gamma_{2}\right)$ can be reduced, by eliminating all the eigenvalues of the linearization of $s_{2}$ around the origin that are not on the imaginary axis, to obtain a local immersion of a lower order. The proof of this is based on the center manifold theorem.

We denote by $I$ and by $\mathcal{B}(w, d)$ the identity operator and the open ball of radius $d>0$ around $w$, respectively, on any space (the space will be clear from the context). We use the concept of spectral projection operator, see [20, p. 32] for details.

Theorem 3.1. Consider an autonomous system $\left(W_{2}, s_{2}, \gamma_{2}\right)$ of order $r_{2}$ and define $S_{2}=\left[\frac{\partial s_{2}}{\partial \zeta}\right]_{(0)}$. Let $\mathbf{P}: \mathbb{R}^{r_{2}} \rightarrow \mathbb{R}^{r_{2}}$ denote the spectral projection operator corresponding to the set of all eigenvalues of $S_{2}$ that lie on the imaginary axis. Let $E_{0}$ and $E_{h}$ be the images of $\mathbf{P}$ and $I-\mathbf{P}$, respectively. Then there exists a $C^{2}$ map $\Psi: E_{0} \rightarrow E_{h}$ with $\Psi(0)=0,\left[\frac{\partial \Psi}{\partial \zeta}\right]_{(0)}=0$ and a ball $\mathcal{B}(0, d) \subset W_{2}$ such that the center manifold

$$
\mathcal{M}_{0}=\left\{w_{0}+\Psi\left(w_{0}\right) \mid w_{0} \in E_{0}\right\} \subset \mathbb{R}^{r_{2}}
$$

has the following properties:

1. $\mathcal{M}_{0}$ is locally invariant, which means the following: for each state trajectory $w_{2}$ of $\left(W_{2}, s_{2}, \gamma_{2}\right)$ satisfying $w_{2}(0) \in \mathcal{M}_{0} \cap \mathcal{B}(0, d)$ and $w_{2}(t) \in \mathcal{B}(0, d)$ for all $t \in[0, T]$, we have $w_{2}(t) \in \mathcal{M}_{0}$ for all $t \in[0, T]$.

2. Every state trajectory $w_{2}$ of $\left(W_{2}, s_{2}, \gamma_{2}\right)$ satisfying $w_{2}(t) \in \mathcal{B}(0, d)$ for all $t \in$ $(-\infty, \infty)$ also satisfies $w_{2}(0) \in \mathcal{M}_{0}$. 
Moreover, for each state trajectory $w_{2}$ of $\left(W_{2}, s_{2}, \gamma_{2}\right)$ satisfying $w_{2}(t) \in \mathcal{M}_{0} \cap$ $\mathcal{B}(0, d)$ for all $t \in[0, T)$, the function $w(t)=\mathbf{P} w_{2}(t)$ satisfies

$$
w_{2}=w+\Psi(w), \quad \frac{\mathrm{d} w}{\mathrm{~d} t}=\mathbf{P} s_{2}(w+\Psi(w)) \quad \forall t \in[0, T) .
$$

Proposition 3.2. Suppose that for the dynamics (2.16) of the autonomous system $\left(W_{1}, s_{1}, \gamma_{1}\right)$, the origin is a Lyapunov stable equilibrium point and every $w_{0} \in W_{1}$ is nonwandering (as defined before (2.5)). Let $\left(W_{1}, s_{1}, \gamma_{1}\right)$ be locally immersed into an autonomous system $\left(W_{2}, s_{2}, \gamma_{2}\right)$ of order $r_{2}$ via the map $\tau$ (as in Definition 2.7). Denote $S_{2}=\left[\frac{\partial s_{2}}{\partial \zeta}\right]_{(0)}$ and $\Gamma_{2}=\left[\frac{\partial \gamma_{2}}{\partial \zeta}\right]_{(0)}$.

Then there exists an autonomous system $\left(W_{3}, s_{3}, \gamma_{3}\right)$ with linearization $S_{3}=$ $\left[\frac{\partial s_{3}}{\partial \zeta}\right]_{(0)}$ and $\Gamma_{3}=\left[\frac{\partial \gamma_{3}}{\partial \zeta}\right]_{(0)}$ such that $\left(W_{1}, s_{1}, \gamma_{1}\right)$ is locally immersed into $\left(W_{3}, s_{3}, \gamma_{3}\right)$, $S_{3}$ is isomorphic to the restriction of $S_{2}$ to one of its invariant subspaces and all the eigenvalues of $S_{3}$ are on the imaginary axis.

Furthermore if the pair $\left(\Gamma_{2}, S_{2}\right)$ is detectable, then so is the pair $\left(\Gamma_{3}, S_{3}\right)$.

Proof. Due to our Lyapunov stability assumption for (2.16), there exists an open set $W_{0} \subset W_{1}$ containing the origin and $\delta>0$ such that $\overline{\mathcal{B}(0,2 \delta)} \subset W_{1}$ and if $w_{1}(0) \in W_{0}$, then for the solution $w_{1}$ of (2.16), $\left\|w_{1}(t)\right\|<\delta$ holds for all $t \geq 0$.

We claim that for all $w_{1}(0) \in W_{0},(\underline{2.16})$ has a well-defined trajectory backwards in time and $\left\|w_{1}(t)\right\|<2 \delta$ for all $t<0$. To prove this, fix $w_{1}(0) \in W_{0}$. Then there exists a maximal interval $(T, 0]$ with $T<0$ on which (2.16) has a unique solution $w_{1}$ and if $|T|<\infty$, then there exists a $t_{0} \in(T, 0)$ such that $\left\|w_{1}\left(t_{0}\right)\right\|>2 \delta$. All this follows by applying the standard results about existence and uniqueness of solutions for ordinary differential equations (ODEs), evolving forward in time, to the ODE $\dot{w}=-s_{1}(w)$ (see, e.g., [30] or [27, Theorem II.2 and Corollary II.3]). Thus, to prove our claim, we have to prove that $\left\|w_{1}\left(t_{0}\right)\right\|>2 \delta$ cannot occur for any $t_{0}<0$.

Suppose that there exists a $t_{0} \in(T, 0)$ with $\left\|w_{1}\left(t_{0}\right)\right\|>2 \delta$. Clearly, $w_{1}$ can be extended uniquely to a solution of (2.16) ) on $(T, \infty)$ (recall that $w(t) \in W_{1}$ for all $t \in$ $(T, \infty)$ and $\left.w_{1}(0) \in W_{0}\right)$. Denote by $\phi$ the flow generated by $s_{1}$ on $W_{1}$. Since every point in $W_{1}$ is nonwandering, according to (2.5) we can find a sequence $\left(w_{n}\right)$ in $W_{1}$ and a sequence $\left(t_{n}\right)$ in $(0, \infty)$ such that $t_{n} \rightarrow \infty, w_{n} \rightarrow w_{1}\left(t_{0}\right)$ and $\phi_{t_{n}}\left(w_{n}\right) \rightarrow w_{1}\left(t_{0}\right)$. According to the continuous dependence of solutions of ODEs on the initial state ([30, Chapter 3]) we have, in addition, that $\phi_{-t_{0}}\left(w_{n}\right) \rightarrow \phi_{-t_{0}}\left(w_{1}\left(t_{0}\right)\right)=w_{1}(0)$. Thus, for $n$ sufficiently large, $\phi_{-t_{0}}\left(w_{n}\right) \in W_{0}$ and hence for $t>0$ the trajectory $\phi_{t-t_{0}}\left(w_{n}\right)$ remains in $\mathcal{B}(0, \delta)$. This contradicts the earlier conclusion that the same trajectory has $w_{1}\left(t_{0}\right)$ as one limit point. Thus we have proved our claim.

We complete this proof by applying the center manifold theorem to $\left(W_{2}, s_{2}, \gamma_{2}\right)$. Recall the open ball $\mathcal{B}(0, d)$, the subspaces $E_{0}, E_{h} \subset \mathbb{R}^{r_{2}}$, the center manifold $\mathcal{M}_{0}$, the nonlinear map $\Psi: E_{0} \rightarrow E_{h}$ and the projector $\mathbf{P}: \mathbb{R}^{r_{2}} \rightarrow E_{0}$ from Theorem 3.1. The system $\left(W_{1}, s_{1}, \gamma_{1}\right)$ is locally immersed into $\left(W_{2}, s_{2}, \gamma_{2}\right)$ via the $C^{2}$ map $\tau$. First we show that the image of any state trajectory of (2.16), starting from some $w_{1}(0) \in W_{0}$, under $\tau$ is contained in $\mathcal{M}_{0}$. For this we suppose that $\delta>0$ 
is sufficiently small such that $\tau \mathcal{B}(0,2 \delta) \subset \mathcal{B}(0, d)$ ( $\delta$ can be made arbitrarily small by choosing $W_{0}$ appropriately). The claim established earlier implies that for each $w_{1}(0) \in W_{0}$, there exists a unique solution $w_{1}: \mathbb{R} \rightarrow W_{1}$ for (2.16) and $\left\|w_{1}(t)\right\|<2 \delta$ for all $t \in \mathbb{R}$. This and $\tau \mathcal{B}(0,2 \delta) \subset \mathcal{B}(0, d)$ imply, via Theorem 3.1, that

$$
\tau w_{1}(t) \in \mathcal{M}_{0} \cap \mathcal{B}(0, d) \quad \forall t \in \mathbb{R} .
$$

Next we construct $\left(W_{3}, s_{3}, \gamma_{3}\right)$ with the desired properties. Denote $r_{3}=\operatorname{dim} E_{0}$ and let $\mathcal{J}$ be the linear isomorphism from $E_{0}$ to $\mathbb{R}^{r_{3}}$. Define $W_{3}=\mathcal{J}\left(E_{0} \cap \mathcal{B}(0, d)\right)$. Clearly $W_{3}$ is an open set containing the origin. For any point $w_{1}(0) \in W_{0}$, (3.2) and (3.1) give that the function $w_{3}=\mathcal{J} \mathbf{P} w_{2}$, where $w_{2}$ is the state trajectory of $\left(W_{2}, s_{2}, \gamma_{2}\right)$ with $w_{2}(0)=\tau w_{1}(0)$, is such that $w_{3}(t) \in W_{3}$ for all $t \in[0, \infty)$ and on this interval

$$
\frac{\mathrm{d} w_{3}}{\mathrm{~d} t}=\mathcal{J} \mathbf{P} s_{2}\left(\mathcal{J}^{-1} w_{3}+\Psi\left(\mathcal{J}^{-1} w_{3}\right)\right) .
$$

This motivates to define the maps $s_{3}: W_{3} \rightarrow \mathbb{R}^{r_{3}}$ and $\gamma_{3}: W_{3} \rightarrow \mathbb{R}$ by

$$
s_{3}(w)=\mathcal{J} \mathbf{P} s_{2}\left(\mathcal{J}^{-1} w+\Psi\left(\mathcal{J}^{-1} w\right)\right), \quad \gamma_{3}(w)=\gamma_{2}\left(\mathcal{J}^{-1} w+\Psi\left(\mathcal{J}^{-1} w\right)\right) .
$$

Clearly $\left(W_{3}, s_{3}, \gamma_{3}\right)$ is an autonomous system of order $r_{3}$. It is easy to check, using the definitions of $\mathbf{P}$ and $\Psi$ and the facts

$$
\tau\left(w_{0}\right) \in \mathcal{M}_{0} \cap \mathcal{B}(0, d), \quad \mathbf{P} \tau\left(w_{0}\right)+\Psi\left(\mathbf{P} \tau\left(w_{0}\right)\right)=\tau\left(w_{0}\right) \quad \forall w_{0} \in W_{0}
$$

that $\left(W_{1}, s_{1}, \gamma_{1}\right)$ is locally immersed in $\left(W_{3}, s_{3}, \gamma_{3}\right)$ via the map $\mathcal{J} \mathbf{P} \tau$. The linearization of $s_{3}$ around the origin is $S_{3}=\mathcal{J} \mathbf{P} S_{2} \mathcal{J}^{-1}$, i.e. $S_{3}$ is isomorphic to the restriction of $S_{2}$ to its invariant subspace $E_{0}$ and $\sigma_{p}\left(S_{3}\right)=\sigma_{p}\left(S_{2}\right) \cap i \mathbb{R}$. This completes the proof of the main claim of the proposition.

The linearization of $\gamma_{3}$ around the origin is $\Gamma_{3}=\Gamma_{2} \mathcal{J}^{-1}$. Assume that the pair $\left(\Gamma_{2}, S_{2}\right)$ is detectable but the pair $\left(\Gamma_{3}, S_{3}\right)$ is not. Then, using the Hautus test (see, for instance, [31, p. 14]), it follows that for some $p \in \mathbb{C}$ with $\operatorname{Re} p \geq 0$, some $w \in \mathbb{R}^{r_{3}}$ and denoting $v=\mathcal{J}^{-1} w \in E_{0}$,

$$
0=\left[\begin{array}{c}
S_{3} w-p w \\
\Gamma_{3} w
\end{array}\right]=\left[\begin{array}{c}
\mathcal{J} \mathbf{P} S_{2} v-p \mathcal{J} v \\
\Gamma_{2} v
\end{array}\right]=\left[\begin{array}{c}
\mathbf{P} S_{2} v-p v \\
\Gamma_{2} v
\end{array}\right] .
$$

To get the last equality we have used the fact that $\mathbf{P} S_{2} v-p v \in E_{0}$ and $\mathcal{J}: E_{0} \rightarrow \mathbb{R}^{r_{3}}$ is an isomorphism. Since $E_{0}$ is an invariant subspace for $S_{2}, v \in E_{0}$ and $\mathbf{P}$ is the projection onto $E_{0}$, we get that $\mathbf{P} S_{2} v-p v=S_{2} v-p v$. This and (3.3) give the contradiction that $\left(\Gamma_{2}, S_{2}\right)$ is not detectable. Thus if $\left(\Gamma_{2}, S_{2}\right)$ is detectable, then $\left(\Gamma_{3}, S_{3}\right)$ must also be detectable.

Remark 3.3. Let $\left(W_{1}, s_{1}, \gamma_{1}\right),\left(W_{2}, s_{2}, \gamma_{2}\right),\left(W_{3}, s_{3}, \gamma_{3}\right), S_{2}$ and $S_{3}$ be as in the above proposition. In particular, $\left(W_{2}, s_{2}, \gamma_{2}\right)$ is a local immersion of order $r_{2}$ for $\left(W_{1}, s_{1}, \gamma_{1}\right)$ and $\left(W_{3}, s_{3}, \gamma_{3}\right)$ is a local immersion of order $r_{3}$ for $\left(W_{1}, s_{1}, \gamma_{1}\right)$. Since $S_{3}$ is isomorphic to the restriction of $S_{2}$ to one of its invariant subspaces, it follows that $r_{3} \leq r_{2}$ and $\sigma_{p}\left(S_{3}\right) \subset \sigma_{p}\left(S_{2}\right)$ and furthermore, since $\sigma_{p}\left(S_{3}\right) \subset i \mathbb{R}$, if $\sigma_{p}\left(S_{2}\right) \not \subset$ $i \mathbb{R}$, then $r_{3}<r_{2}$. Hence if $\left(W_{2}, s_{2}, \gamma_{2}\right)$ is a local immersion of smallest order for $\left(W_{1}, s_{1}, \gamma_{1}\right)$, then clearly $r_{3}<r_{2}$ cannot occur and so $\sigma_{p}\left(S_{2}\right) \subset i \mathbb{R}$. 


\section{A minimal order controller}

Using the notation and assumptions from Subsection 2.1, in this section we construct a low order controller that solves the local error feedback regulator problem. Under some technical assumptions this controller is of minimal order. This controller can be viewed as a nonlinear version of the controller (2.20). Our main theorem below is complicated, but if we are willing to compromise a bit on the generality, then we get a much more simple version of it, stated below as Corollary 4.3. The theorem states the existence of the desired low order controller, but we also provide a way to construct such a controller, see Remark 4.4.

Theorem 4.1. Suppose that $A$ is stable and there exist $C^{2}$ maps $\pi: W^{o} \rightarrow X$ and $\gamma: W^{o} \rightarrow U$, where $W^{o} \subset W$ is an open set containing zero, that satisfy the nonlinear regulator equations (2.17) and (2.18), with $\pi(0)=0$ and $\gamma(0)=0$. Let the autonomous system $\left\{W^{o}, s, \gamma\right\}$ be locally immersed into the autonomous system $\left\{E^{o}, \phi, \lambda\right\}$, where $E^{o} \subset \mathbb{R}^{\nu}$ is an open set containing zero and $\phi: E^{o} \rightarrow$ $\mathbb{R}^{\nu}$ and $\lambda: E^{o} \rightarrow \mathbb{R}$ are $C^{2}$ maps satisfying $\phi(0)=0$ and $\lambda(0)=0$. Assume that the linearizations $\Phi=\left[\frac{\partial \phi}{\partial \zeta}\right]_{(0)}$ and $\Lambda=\left[\frac{\partial \lambda}{\partial \zeta}\right]_{(0)}$ satisfy the following conditions:

$$
(\Lambda, \Phi) \text { is detectable, } \quad \mathbf{G}(p) \neq 0 \quad \forall p \in \sigma_{p}(\Phi) \cap i \mathbb{R} \text {. }
$$

If $\sigma_{p}(\Phi) \subset i \mathbb{R}$, then there exists a $B_{c} \in \mathbb{R}^{\nu}$ such that the controller

$$
\dot{\xi}=\phi(\xi)+B_{c} e, \quad u=\lambda(\xi),
$$

solves the local error feedback regulator problem for the plant (2.1), the exosystem (2.2) and the error (2.4).

If $\sigma_{p}(\Phi) \not \subset i \mathbb{R}$, then there is a local immersion $\left\{E_{s}^{o}, \phi_{s}, \lambda_{s}\right\}$ of smaller order for $\left\{W^{o}, s, \gamma\right\}$, i.e. $E_{s}^{o} \subset \mathbb{R}^{\nu_{s}}$ with $\nu_{s}<\nu$, such that denoting $\Phi_{s}=\left[\frac{\partial \phi_{s}}{\partial \zeta}\right]_{(0)}$ and $\Lambda_{s}=\left[\frac{\partial \lambda_{s}}{\partial \zeta}\right]_{(0)}$, the pair $\left(\Lambda_{s}, \Phi_{s}\right)$ is detectable and $\sigma_{p}\left(\Phi_{s}\right) \subset \sigma_{p}(\Phi) \cap i \mathbb{R}$. In this case, there exists $B_{c s} \in \mathbb{R}^{\nu_{s}}$ such that the controller

$$
\dot{\xi}=\phi_{s}(\xi)+B_{c s} e, \quad u=\lambda_{s}(\xi),
$$

solves the local error feedback regulator problem for the plant (2.1), the exosystem (2.2) and the error (2.4).

Let $\nu>0$ be the smallest integer such that there exist some $\pi$ and $\gamma$ as above and a local immersion $\left\{E^{o}, \phi, \lambda\right\}$ of order $\nu$ for $\left\{W^{o}, s, \gamma\right\}$, with linearizations $\Phi=\left[\frac{\partial \phi}{\partial \zeta}\right]_{(0)}$ and $\Lambda=\left[\frac{\partial \lambda}{\partial \zeta}\right]_{(0)}$, for which the conditions in (4.1) hold. Then $\sigma_{p}(\Phi) \subset i \mathbb{R}$ and the corresponding controller (4.2) of order $\nu$ is minimal, i.e. it is of the lowest possible order among controllers of the form (2.9) that solve this error feedback regulator problem.

Remark 4.2. Theorem 4.1 can be regarded as a refinement/extension of Theorem 2.8 when the plant is locally exponentially stable. Following Theorem 2.8, the above 
theorem assumes that the nonlinear regulator equations (2.17) and (2.18) are solved by functions $\pi$ and $\gamma$ defined on $W^{o}$ and that $\left(W^{o}, s, \gamma\right)$ is locally immersed into the internal model $\left(E^{o}, \phi, \lambda\right)$. But instead of the condition (2.19) in Theorem 2.8 we assume in Theorem 4.1 that (4.1) holds, which is implied by (2.19). In the linear SISO case, the internal model can be chosen to be a copy of the exosystem, but in the nonlinear SISO case this is not always possible, see Example 5.2 .

Proof. Consider the unforced closed-loop system of the plant (2.1) and the controller (4.2) (unforced meaning $w=0$ and so $y=e$ ). The state operator of the linearization of this closed-loop system at the origin is

$$
\mathcal{A}_{c l}=\left[\begin{array}{cc}
A & B \Lambda \\
B_{c} C & \Phi+B_{c} D \Lambda
\end{array}\right] .
$$

Suppose that (4.1) holds and $\sigma_{p}(\Phi) \subset i \mathbb{R}$. It then follows from Proposition 2.10 that there exists a $B_{c} \in \mathbb{R}^{\nu}$ such that $\mathcal{A}_{c l}$ is exponentially stable. Fix such a $B_{c}$. The forced closed-loop system of the plant (2.1) and the controller (4.2) (with $B_{c}$ chosen as above), with the error (2.4) as its output, is

$$
\left[\begin{array}{c}
\dot{x} \\
\dot{\xi}
\end{array}\right]=\mathcal{F}(x, \xi, w), \quad e=h(x, \gamma(\xi), w),
$$

where

$$
\mathcal{F}(x, \xi, w)=\left[\begin{array}{c}
f(x, \gamma(\xi), w) \\
\phi(\xi)+B_{c} h(x, \gamma(\xi), w)
\end{array}\right] .
$$

This closed-loop system is locally exponentially stable when $w$ is identically zero, since the linearization of $\mathcal{F}(x, \xi, 0)$ at $(x, \xi)=(0,0)$ (its Jacobian) is $\mathcal{A}_{c l}$.

We will now apply Lemma 2.4 to show that the controller (4.2) solves the local error feedback regulator problem for the plant (2.1), the exosystem (2.2) and the error (2.4). Comparing (4.2) to its general form (2.9) we get

$$
\eta(\xi, 0)=\phi(\xi), \quad \theta(\xi)=\lambda(\xi) \quad \forall \xi \in E^{o} .
$$

Let $\tau: \widetilde{W}^{o} \rightarrow E^{o}$ be a $C^{2}$ map that locally immerses $\left\{W^{o}, s, \gamma\right\}$ into $\left\{E^{o}, \phi, \lambda\right\}$. Here $\widetilde{W^{o}} \subset W^{o}$ is a open set containing zero. So $\tau(0)=0$,

$$
\frac{\partial \tau}{\partial w} s(w)=\phi(\tau(w)), \quad \gamma(w)=\lambda(\tau(w)) \quad \forall w \in \widetilde{W}^{0} .
$$

By assumption, the maps $\pi: W^{o} \rightarrow X$ and $\gamma: W^{o} \rightarrow U$ satisfy the nonlinear regulator equations (2.17) and (2.18). It follows from this, (4.5) and (4.6) that if we define $\sigma(w)=\tau(w)$ for all $w \in \widetilde{W}^{o}$, then the pair $(\pi, \sigma)$ satisfy (2.12)-(2.14) (with $\widetilde{W}^{o}$ in place of $W^{o}$ in Lemma 2.4). Thus the controller (4.2) solves the above error feedback regulator problem.

If $\sigma_{p}(\Phi) \not \subset i \mathbb{R}$, then the existence of a local immersion $\left\{E_{s}^{o}, \phi_{s}, \lambda_{s}\right\}$ for $\left\{W^{o}, s, \gamma\right\}$, with the properties described in the theorem, follows directly from Proposition 3.2 . In this case, a $B_{c s} \in \mathbb{R}^{\nu_{s}}$ such that the controller (4.3) solves the local error feedback regulator problem under consideration can be found by applying the first part of this theorem to $\left\{E_{s}^{o}, \phi_{s}, \lambda_{s}\right\}$ in place of $\left\{E^{o}, \phi, \lambda\right\}$. 
Finally, suppose that $\nu>0$ is the smallest integer such that for some $\pi$ and $\gamma$ as in the theorem, there is a local immersion $\left\{E^{o}, \phi, \lambda\right\}$ of order $\nu$ for $\left\{W^{o}, s, \gamma\right\}$ for which (4.1) holds. Fix such $\pi, \gamma$ and $\left\{E^{o}, \phi, \lambda\right\}$ of order $\nu$. Our assumptions on the exosystem in (2.2) imply that for the dynamics of $\left\{W^{o}, s, \gamma\right\}$, the origin is a Lyapunov stable equilibrium point and every point in $W^{o}$ is nonwandering. Since $\left\{E^{o}, \phi, \lambda\right\}$ is a local immersion of the smallest order for $\left\{W^{o}, s, \gamma\right\}$, it follows from Proposition 3.2 (see also Remark 3.3 ) that $\sigma_{p}(\Phi) \subset i \mathbb{R}$. Hence, $B_{c}$ can be found so that the controller (4.2) solves the local error feedback regulator problem. We claim that this controller is minimal. In the remaining part of this proof, we establish this claim by contradiction.

Assume that a controller $\mathbf{C}$ of the form (2.9), determined by the maps $\eta$ and $\theta$, with order $n_{c}<\nu$ solves the local error feedback regulator problem. The state of this controller belongs to an open set $X_{c} \subset \mathbb{R}^{n_{c}}$ containing zero . Define $\psi(\xi)=\eta(\xi, 0)$ for all $\xi \in X_{c}$. It now follows from Lemma 2.4 that there exist $C^{2}$ maps $\tilde{\pi}: W^{1} \rightarrow X$ and $\tilde{\sigma}: W^{1} \rightarrow X_{c}$, where $W^{1} \subset W^{o}$ is open and contains zero, such that $\tilde{\pi}(0)=0$ and $\tilde{\sigma}(0)=0$ and (2.12)-(2.14) hold (with $\tilde{\pi}, \tilde{\sigma}$ in place of $\pi, \sigma$ and $W^{1}$ in place of $\left.W^{o}\right)$. Define $\tilde{\gamma}(w)=\theta(\tilde{\sigma}(w))$ for all $w \in W^{1}$. Comparing (2.12) with (2.17) and (2.14) with (2.18) it follows that $\tilde{\pi}$ and $\tilde{\gamma}$ solve the regulator equations (2.17) $-(2.18)$. This and (2.13) imply that $\left\{X_{c}, \psi, \theta\right\}$ is a local immersion for $\left\{W^{o}, s, \tilde{\gamma}\right\}$. Recall the matrices $F, G$ and $K$ from (2.10) that determine the linearized controller. The state operator for the linearization of the unforced closed-loop system of the plant (2.1) and the controller $\mathbf{C}$ at the origin is

$$
\mathcal{A}_{f}=\left[\begin{array}{cc}
A & B K \\
G C & F+G D K
\end{array}\right]
$$

which by assumption is stable. This means that the linearized controller $(F, G, K)$ is stabilized by the linearized plant $(A, B, C, D)$. Therefore $(K, F)$ is detectable and there is no unstable pole/zero cancelations in the product of the plant and the controller transfer functions. This implies that $\mathbf{G}(p) \neq 0$ for each $p \in \sigma_{p}(F) \cap i \mathbb{R}$. We get that $\left\{X_{c}, \psi, \theta\right\}$ is a local immersion of order $n_{c}<\nu$ for $\left\{W^{o}, s, \tilde{\gamma}\right\}$ for which (4.1) holds (with $(K, F)$ in place of $(\Lambda, \Phi)$ ). But this contradicts the minimality of $\nu$ assumed in the last part of the theorem.

Recall the matrices $A, P, S, C$ and $Q$ defined below (2.6). When the pair of matrices $\left(\left[\begin{array}{ll}C & Q\end{array}\right],\left[\begin{array}{ll}A & P \\ 0 & S\end{array}\right]\right)$ is detectable, we have the following corollary of Theorem 4.1 .

Corollary 4.3. Suppose that $A$ is stable, the matrix pair $\left(\left[\begin{array}{ll}C & Q\end{array}\right],\left[\begin{array}{ll}A & P \\ 0 & S\end{array}\right]\right)$ is detectable and there exist $C^{2}$ maps $\pi: W^{o} \rightarrow X$ and $\gamma: W^{o} \rightarrow U$, where $W^{o} \subset W$ is an open set containing zero, that satisfy the nonlinear regulator equations (2.17) and (2.18), with $\pi(0)=0$ and $\gamma(0)=0$. Then there exists a $B_{c} \in \mathbb{R}^{p}$ such that the controller

$$
\dot{\xi}=s(\xi)+B_{c} e, \quad u=\gamma(\xi),
$$

solves the local error feedback regulator problem for the plant (2.1), the exosystem (2.2) and the error (2.4). Moreover, this controller is minimal, i.e. it is of the lowest possible order among all the controllers of the form (2.9) that solve this error feedback regulator problem. 
Proof. Since $\pi$ and $\gamma$ satisfy the nonlinear regulator equations (2.17) and (2.18) (and $\pi(0)=0$ and $\gamma(0)=0$ ), by linearizing these equations at $w=0$, we get

$$
\Pi S=A \Pi+B \Gamma+P, \quad C \Pi+D \Gamma+Q=0,
$$

where $\Pi=\left[\frac{\partial \pi}{\partial w}\right]_{w=0}$ and $\Gamma=\left[\frac{\partial \gamma}{\partial w}\right]_{w=0}$. Since the the pair $\left(\left[\begin{array}{ll}C & Q\end{array}\right],\left[\begin{array}{ll}A & P \\ 0 & S\end{array}\right]\right)$ is detectable, it follows from the Hautus test that

$$
\operatorname{Ker}\left[\begin{array}{cc}
A-i \alpha I & P \\
0 & S-i \alpha I \\
C & Q
\end{array}\right]=\left\{\left[\begin{array}{l}
0 \\
0
\end{array}\right]\right\} \quad \forall i \alpha \in \sigma_{p}(S) .
$$

First we show that the pair $(\Gamma, S)$ is detectable. Suppose that $(\Gamma, S)$ is not detectable. It then follows using the Hautus test that for some $i \alpha \in \sigma_{p}(S)$ and some $d \in \mathbb{C}^{p}$ with $d \neq 0,(S-i \alpha I) d=0$ and $\Gamma d=0$. Using these expressions, by multiplying both the equations in (4.9) with $d$ (from the right) we get that

$$
(A-i \alpha I) \Pi d+P d=0, \quad C \Pi d+Q d=0 .
$$

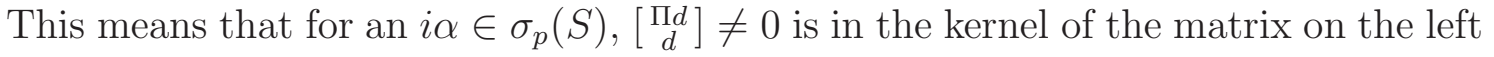
of (4.10), which contradicts (4.10). Hence $(\Gamma, S)$ must be a detectable pair. This implies that the geometric multiplicity of each eigenvalue of $S$ is 1 .

Next we show that $\mathbf{G}(i \alpha) \neq 0$ for all $i \alpha \in \sigma_{p}(S)$. Suppose that $\mathbf{G}(i \alpha)=0$ for some $i \alpha \in \sigma_{p}(S)$. Fix $d \in \mathbb{C}^{p}$ non-zero such that $(S-i \alpha I) d=0$. From (4.9) we get

$$
(A-i \alpha I) \Pi d+B \Gamma d+P d=0, \quad C \Pi d+D \Gamma d+Q d=0 .
$$

Solving for $\Pi d$ from the first equation above and substituting for it in the second equation, and then using $\mathbf{G}(i \alpha)=0$, we get that $-C(A-i \alpha I)^{-1} P d+Q d=0$. This means that $\left[\begin{array}{c}-(A-i \alpha I)^{-1} P d \\ d\end{array}\right] \neq 0$ is in the kernel of the matrix on the left of (4.10) for some $i \alpha \in \sigma_{p}(S)$, which contradicts (4.10). Hence $\mathbf{G}(i \alpha) \neq 0$ for all $i \alpha \in \sigma_{p}(S)$. Thus (4.1) holds (with $(\Gamma, S)$ in place of $(\Lambda, \Phi)$ ). It now follows from Theorem 4.1 that there exists $B_{c} \in \mathbb{R}^{p}$ such that the controller (4.8) solves the local error feedback regulator problem for the plant (2.1), the exosystem (2.2) and the error (2.4).

To complete the proof of this corollary, we must show that the controller (4.8) is minimal. For this, according to Theorem 4.1, it is sufficient to show that $\left(W^{o}, s, \gamma\right)$, independently of our choice of $\pi$ and $\gamma$ that solve (2.17) and (2.18), cannot be locally immersed into an autonomous system $\left(E^{o}, \phi, \lambda\right)$ of lower order. Suppose that $\left(W^{o}, s, \gamma\right)$ is locally immersed in $\left(E^{o}, \phi, \lambda\right)$ via the map $\tau$, whose linearization at the origin is $T$. Let $\Phi$ and $\Lambda$ be linearizations of $\phi$ and $\lambda$ at the origin. Then (4.6) holds and linearizing it around $w=0$ gives

$$
T S=\Phi T, \quad \Gamma=\Lambda T .
$$

Let $i \alpha$ be an eigenvalue of $S$ with algebraic multiplicity $k$. Then for some $d \in \mathbb{C}^{p}$

$$
(S-i \alpha I)^{k-1} d \neq 0, \quad(S-i \alpha I)^{k} d=0 .
$$

Since $(\Gamma, S)$ is a detectable pair, using the Hautus test and $(S-i \alpha I)^{k} d=0$, we get that $\Gamma(S-i \alpha I)^{k-1} d \neq 0$. The first equation in (4.11) implies that $T(S-\mu I)^{m}=$ $(\Phi-\mu I)^{m} T$ for each $\mu \in \mathbb{C}$ and any integer $m \geq 0$. Hence, using (4.12), we have 


$$
0=T(S-i \alpha I)^{k} d=(\Phi-i \alpha I)^{k} T d, \quad T(S-i \alpha I)^{k-1} d=(\Phi-i \alpha I)^{k-1} T d .
$$

The second equation above, using $\Gamma=\Lambda T$ from (4.11) and $\Gamma(S-i \alpha I)^{k-1} d \neq 0$, gives $\Lambda(\Phi-i \alpha I)^{k-1} T d \neq 0$. Therefore $(\Phi-i \alpha I)^{k-1} T d \neq 0$ and $(\Phi-i \alpha I)^{k} T d=0$ (see the first equation above). This means that $i \alpha$ is an eigenvalue of $\Phi$ with algebraic multiplicity $m \geq k$. Thus we have shown that if $i \alpha$ is an eigenvalue of $S$ with algebraic multiplicity $k$, then it is also an eigenvalue of $\Phi$ with algebraic multiplicity $m$ with $m \geq k$. Hence the order of $\left(E^{o}, \phi, \lambda\right)$ must be larger than or equal to the order of $\left(W^{o}, s, \gamma\right)$. Thus the controller (4.8) is minimal.

Remark 4.4. While Theorem 4.1 and Corollary 4.3 only state the existence of low order controllers that solve the local error feedback regulator problem, their proofs contain a method for constructing such controllers. In Theorem 4.1, suppose that $\sigma_{p}(\Phi) \subset i \mathbb{R}$. Then (4.2) is the required controller in which $\phi$ and $\lambda$ are known (given) and $B_{c}$ should be chosen so that $\mathcal{A}_{c l}$ in (4.4) is stable. Remark 2.11 (see also Proposition 2.10) describes a method for choosing such a $B_{c}$. When $\sigma_{p}(\Phi) \not \subset i \mathbb{R}$, a lower order immersion must be constructed as described in the proof of Proposition 3.2 (this is a nontrivial task). Then (4.3) is the required controller in which $B_{c}$ should be chosen so that $\mathcal{A}_{c l}$ in (4.4), with $\left(\Phi_{s}, \Lambda_{s}\right)$ in place of $(\Phi, \Lambda)$, is stable. Similarly in Corollary 4.3, (4.8) is the required controller in which the unknown $B_{c}$ should be chosen so that $\mathcal{A}_{c l}$ in (4.4), with $(S, \Gamma)$ in place of $(\Phi, \Lambda)$, is stable.

Remark 4.5. In this section we have assumed that the state operator $A$ of the linearized plant is stable. As evident from the proof of Theorem 4.1, this assumption is used only to guarantee the existence of a vector $B_{c} \in \mathbb{R}^{\nu}$ such that $\mathcal{A}_{c l}$ in (4.4) is stable. Therefore, instead of the stability of $A$, we could have directly assumed the existence of such a vector, which is a more general assumption. For example, if

$$
\begin{gathered}
A=\left[\begin{array}{cc}
0 & 1 \\
1 & -1
\end{array}\right], \quad B=\left[\begin{array}{c}
0 \\
10
\end{array}\right], \quad C=\left[\begin{array}{ll}
3 & 4
\end{array}\right], \quad D=0, \\
\Phi=\left[\begin{array}{cc}
0 & 1 \\
-1 & 0
\end{array}\right], \quad \Lambda=\left[\begin{array}{ll}
-4 & -5
\end{array}\right],
\end{gathered}
$$

then $A$ is unstable but $B_{c}=\left[\begin{array}{l}1 \\ 2\end{array}\right]$ ensures that $\mathcal{A}_{c l}$ is stable. The problem of finding $B_{c}$ such that $\mathcal{A}_{c l}$ is stable is equivalent to the static output feedback stabilization problem for the following linear time invariant plant:

$$
\left[\begin{array}{l}
\dot{z}_{1} \\
\dot{z}_{2}
\end{array}\right]=\left[\begin{array}{cc}
A^{\top} & 0 \\
\Lambda^{\top} B^{\top} & \Phi^{\top}
\end{array}\right]\left[\begin{array}{l}
z_{1} \\
z_{2}
\end{array}\right]+\left[\begin{array}{c}
C^{\top} \\
\Lambda^{\top} D
\end{array}\right] u_{c}, \quad y_{c}=z_{2} .
$$

Here $u_{c}(t) \in \mathbb{R}$ is the input and $y_{c}(t) \in \mathbb{R}^{\nu}$ is the output. Clearly if the static output feedback $u_{c}=K_{c} y_{c}$ stabilizes the above plant, then $B_{c}=K_{c}^{\top}$ ensures that $\mathcal{A}_{c l}$ is stable. For details on the static output feedback stabilization problem, along with some synthesis algorithms, see [9, 17, 44, 47] and the references therein.

Remark 4.6. The claims in Theorem 4.1 and Corollary 4.3 regarding the minimality of certain controllers are valid under our $C^{2}$ smoothness assumption for all the maps 
in this paper. But if we drop the requirement that the controller be described by smooth maps, then there may exist controllers with lower order than those we have proposed. For instance, consider the following plant with state $x(t) \in \mathbb{R}$ and input $u(t) \in \mathbb{R}:$

$$
\dot{x}(t)=-x(t)+w_{1}(t)+u(t) .
$$

Here the disturbance signal $w_{1}(t) \in \mathbb{R}$ is generated by the second-order exosystem

$$
\left[\begin{array}{l}
\dot{w}_{1}(t) \\
\dot{w}_{2}(t)
\end{array}\right]=\left[\begin{array}{cc}
0 & 1 \\
-1 & 0
\end{array}\right]\left[\begin{array}{l}
w_{1}(t) \\
w_{2}(t)
\end{array}\right] .
$$

The tracking error is $e(t)=x(t)$, which is available for feedback. According to Corollary 4.3, the order of any $C^{2}$ controller that solves the local error feedback regulator problem for the above plant, exosystem and error cannot be less than 2 . However, note that the nonlinear discontinuous static output feedback control law $u=-\operatorname{sign} x$ ensures that if $\left|w_{1}(0)\right|+\left|w_{2}(0)\right|<1$, then $\lim _{t \rightarrow \infty} x(t)=0$ for all $x(0) \in \mathbb{R}$. In fact, $x(t)$ converges to 0 in finite time.

\section{$5 \quad$ Examples}

The first example illustrates our controller design approach using a locally stable nonlinear plant and a Lyapunov stable nonlinear exosystem whose state trajectories are all periodic functions. An interesting feature of this exosystem is that the state operator of its linearization at the origin has a nontrivial Jordan block. This example does not require the construction of an immersion. Our second example considers a second order nonlinear plant and a second-order linear exosystem with eigenvalues at $\pm i$. We show that no second-order controller of the form (2.9) can solve the regulator problem for this plant and exosystem. We then construct a third order immersion to solve the regulator problem. In these two examples the regulator equations can be solved by easy algebraic manipulations. The third example is based on a practical problem in power electronics and here the regulator equations reduce to a quasilinear PDE of first order, without boundary conditions. This is a challenging problem that gives us some insight into the nature of the regulator equations. Numerical solutions to the regulator equations have been studied using various approaches (fixed point theorems and iterations, power series, finite elements). The relevant literature is not large, see [1, 5, 6, 21, 42, 43] and the references therein. Our approach in this example is predominantly analytical.

Example 5.1. Consider the two-dimensional nonlinear exosystem

$$
\dot{w}_{1}=w_{2}-w_{1}^{4}, \quad \dot{w}_{2}=-w_{1}^{3} .
$$

The state operator of the linearization of this exosystem at the origin in $\mathbb{R}^{2}$ is $S=$ $\left[\begin{array}{ll}0 & 1 \\ 0 & 0\end{array}\right]$, which is a nontrivial Jordan block. We show that $0 \in \mathbb{R}^{2}$ is a Lyapunov stable equilibrium point for (5.1) and that all the trajectories of (5.1) starting sufficiently near 0 are periodic. This means that (5.1) satisfies all the conditions required of an exosystem, as discussed in Subsection 2.1. Existence and uniqueness of solutions to (5.1) on maximal time intervals follows from the standard theory of ODEs. 
The phase portrait of (5.1) (disregarding the direction along the state trajectories) is symmetric about the vertical axis. Indeed, if $\left(w_{1}, w_{2}\right)$ is a trajectory of (5.1) on some time interval $[0, T]$, then so is $\left(-\tilde{w}_{1}, \tilde{w}_{2}\right)$, where $\tilde{w}_{1}(t)=w_{1}(T-t)$ and $\tilde{w}_{2}(t)=w_{2}(T-t)$ for all $t \in[0, T]$. We claim that each state trajectory of (5.1) with initial condition $\left(0, w_{2}(0)\right)$ for some $w_{2}(0) \in(0,1]$ is periodic.

To establish the above claim, we consider a state trajectory $\left(w_{1}, w_{2}\right)$ of (15.1) with initial condition $\left(0, w_{2}(0)\right)$, where $w_{2}(0) \in(0,1]$. Using the differential equations in (5.1) we can infer the following: $w_{1}(t)$ increases and $w_{2}(t)$ decreases along the state trajectory on some time interval $\left[0, t_{1}\right]$, where $t_{1}$ is such that $w_{1}\left(t_{1}\right) \in(0,1)$, $w_{2}\left(t_{1}\right) \in(0,1)$ and $w_{2}\left(t_{1}\right)=w_{1}^{4}\left(t_{1}\right)$. This means that $w_{2}\left(t_{1}\right)<w_{1}\left(t_{1}\right)$. Suppose that the state trajectory exists and remains in the open first quadrant on a maximal time interval $\left(0, t_{2}\right)$. Then for all $t \in\left(t_{1}, t_{2}\right), w_{2}(t)<w_{2}\left(t_{1}\right)$ (because $\dot{w}_{2}(t)<0$ when $w_{1}(t)>0$ ) and $w_{1}(t)<w_{1}\left(t_{1}\right)$ (because when $w_{2}(t) \in\left(0, w_{2}\left(t_{1}\right)\right)$, then $\dot{w}_{1}(t)<0$ if $\left.w_{1}(t) \geq w_{1}\left(t_{1}\right)\right)$. Hence on the time interval $\left(t_{1}, t_{2}\right),\left(w_{1}, w_{2}\right)$ is confined to the box $\left\{(p, q) \mid p \in\left(0, w_{1}\left(t_{1}\right)\right), q \in\left(0, w_{2}\left(t_{1}\right)\right)\right\}$. Along the state trajectory in this box $\dot{w}_{2}(t)<0$ and $\dot{w}_{2}(t)<\dot{w}_{1}(t)$. This, combined with $w_{2}\left(t_{1}\right)<w_{1}\left(t_{1}\right)$, implies that

$w_{1}(t)=w_{1}\left(t_{1}\right)+\int_{t_{1}}^{t} \dot{w}_{1}(\tau) \mathrm{d} \tau \geq w_{1}\left(t_{1}\right)+\int_{t_{1}}^{t} \dot{w}_{2}(\tau) \mathrm{d} \tau \geq w_{1}\left(t_{1}\right)-w_{2}\left(t_{1}\right) \quad \forall t \in\left(t_{1}, t_{2}\right)$.

Hence $t_{2}<\infty, w_{1}\left(t_{2}\right)>0$ and $w_{2}\left(t_{2}\right)=0$. Since $\dot{w}_{1}(t)<w_{2}(t)$ and $\dot{w}_{2}(t)<0$ whenever $\left(w_{1}(t), w_{2}(t)\right)$ is in the open fourth quadrant and since $\dot{w}_{2}\left(t_{2}\right)<0$, it follows that there exists $t_{3}>t_{2}$ such that on the time interval $\left(t_{2}, t_{3}\right)$ the state trajectory remains in the fourth quadrant, $w_{1}\left(t_{3}\right)=0$ and $w_{2}\left(t_{3}\right)<0$. Now using the symmetry in the phase portrait of (5.1), we can conclude that $\left(w_{1}\left(2 t_{3}-t_{2}\right), w_{2}\left(2 t_{3}-t_{2}\right)\right)=$ $\left(-w_{1}\left(t_{2}\right), 0\right)$ and $\left(w_{1}\left(2 t_{3}\right), w_{2}\left(2 t_{3}\right)\right)=\left(0, w_{2}(0)\right)$, i.e the state trajectory is periodic with period $2 t_{3}$. This completes the proof of the claim.

Next we will show that along the periodic trajectory $\left(w_{1}, w_{2}\right)$ described above,

$$
\max _{t \in\left[0,2 t_{3}\right]}\left|w_{1}(t)\right| \leq\left(w_{2}(0)\right)^{0.25}, \quad \max _{t \in\left[0,2 t_{3}\right]}\left|w_{2}(t)\right|=w_{2}(0) .
$$

From the discussion in the above paragraph we get that $\max _{t \in\left[0,2 t_{3}\right]}\left|w_{1}(t)\right| \leq w_{1}\left(t_{1}\right)$, $w_{1}\left(t_{1}\right)=\left(w_{2}\left(t_{1}\right)\right)^{0.25}$ and $w_{2}\left(t_{1}\right)<w_{2}(0)$. These expressions imply the first inequality in (5.2). We will now show that $\left|w_{2}\left(t_{3}\right)\right|<w_{2}(0)$ which implies the second equality in (5.2). Define

$$
\begin{gathered}
z_{1}(t)=-w_{1}\left(t_{1}-t\right), \quad z_{2}(t)=w_{2}\left(t_{1}-t\right) \quad \forall t \in\left[0, t_{1}\right], \\
\tilde{z}_{1}(t)=-w_{1}\left(t+t_{1}\right), \quad \tilde{z}_{2}(t)=-w_{2}\left(t+t_{1}\right) \quad \forall t \in\left[0, t_{3}-t_{1}\right] .
\end{gathered}
$$

Then $z_{1}(0)=\tilde{z}_{1}(0), z_{2}(0)=-\tilde{z}_{2}(0), z_{1}\left(t_{1}\right)=\tilde{z}_{1}\left(t_{3}-t_{1}\right)=0, z_{2}\left(t_{1}\right)=w_{2}(0)$ and $\tilde{z}_{2}\left(t_{3}-t_{1}\right)=-w_{2}\left(t_{3}\right)$. The functions $z_{1}, z_{2}, \tilde{z}_{1}$ and $\tilde{z}_{2}$ satisfy the equations

$$
\dot{z}_{1}=z_{2}-z_{1}^{4}, \quad \dot{z}_{2}=-z_{1}^{3}, \quad \dot{\tilde{z}}_{1}=\tilde{z}_{2}+\tilde{z}_{1}^{4}, \quad \dot{\tilde{z}}_{2}=-\tilde{z}_{1}^{3}
$$

on their intervals of definition. The curves $\left(z_{1}, z_{2}\right)$ and $\left(\tilde{z}_{1}, \tilde{z}_{2}\right)$ (regarded as state trajectories) are in the closed left half-plane of the phase plane. The curve $\left(z_{1}, z_{2}\right)$ starts vertically above the curve $\left(\tilde{z}_{1}, \tilde{z}_{2}\right)$ in the phase plane and both the curves end on the vertical axis. Suppose that $z_{2}\left(t_{1}\right)<\tilde{z}_{2}\left(t_{3}-t_{1}\right)$. Then these curves 
must intersect at some point $\left(z_{10}, z_{20}\right)$ in the second quadrant of the phase plane such that $z_{10}<0$ and at that point $\mathrm{d} z_{2} / \mathrm{d} z_{1} \leq \mathrm{d} \tilde{z}_{2} / \mathrm{d} \tilde{z}_{1}$. But this inequality is equivalent to the condition $\left|z_{10}\right|^{3} /\left(z_{20}-\left|z_{10}\right|^{4}\right) \leq\left|z_{10}^{3}\right| /\left(z_{20}+\left|z_{10}\right|^{4}\right)$, which holds only if $z_{10}=0$ (here we have used the fact that $w_{2}(t)-w_{1}^{4}(t)>0$ for all $t \in\left[0, t_{1}\right)$ ). Thus $z_{2}\left(t_{1}\right) \geq \tilde{z}_{2}\left(t_{3}-t_{1}\right)$ or equivalently $\left|w_{2}\left(t_{3}\right)\right|<w_{2}(0)$.

Denote the open set enclosed by the periodic state trajectory of (5.1) passing through the point $(0,1)$ by $\Omega$. Fix some $\left(w_{10}, w_{20}\right) \in \Omega$ with $\left(w_{10}, w_{20}\right) \neq(0,0)$. It then follows from (5.2) that there exists a periodic trajectory of (5.1) passing through $(0, q)$, with $q>0$ sufficiently small, such that $\left(w_{10}, w_{20}\right) \notin \Omega_{1}$. Here $\Omega_{1}$ is the open set enclosed by the state trajectory through $(0, q)$. Therefore the trajectory $\left(w_{1}, w_{2}\right)$ of (5.1) starting from $\left(w_{10}, w_{20}\right)$ lies inside the set $\Omega$ and cannot enter the set $\Omega_{1}$ which contains $(0,0)$. This and the second equation in (5.1) imply that the state trajectory $\left(w_{1}, w_{2}\right)$ must come arbitrarily close to the vertical axis in the phase-plane infinitely often which, along with the first equation in (5.1), means that $w_{1}$ must change sign infinitely often and so $\left(w_{1}, w_{2}\right)$ passes through a point $\left(0, \tilde{w}_{20}\right)$ for some $\tilde{w}_{20} \in(0,1)$. From the claim established earlier, and the uniqueness of solutions to (5.1) backwards in time, it follows that the trajectory $\left(w_{1}, w_{2}\right)$ is periodic.

We have shown that all the trajectories starting inside the open set $\Omega$ containing the origin are periodic, i.e. every point $w_{0} \in \Omega$ is nonwandering for the dynamics of the exosystem (5.1). (We remark that it is possible to show that all the trajectories of (5.1) are periodic.) It follows from (5.2) that the origin is a Lyapunov stable equilibrium point for the exosystem (5.1). Below we consider an output regulation problem using this exosystem.

Consider the nonlinear plant

$$
\dot{x}_{1}=x_{2}-w_{1}, \quad \dot{x}_{2}=-x_{1}-x_{2}-\sin \left(x_{2}\right)+\left(1+x_{2}^{2}\right) u
$$

with output $y=x_{1}$. The disturbance signal $\left[\begin{array}{l}w_{1} \\ w_{2}\end{array}\right]$ is generated by the exosystem (5.1). The control objective is to ensure that $\lim _{t \rightarrow \infty} y(t)=0$. Hence in this example the error is $e=x_{1}$ and the linearization in (2.7) and (2.8) are determined by

$$
\begin{array}{lll}
A=\left[\begin{array}{cc}
0 & 1 \\
-1 & -2
\end{array}\right], & B=\left[\begin{array}{l}
0 \\
1
\end{array}\right], & P=\left[\begin{array}{cc}
-1 & 0 \\
0 & 0
\end{array}\right], \\
C=\left[\begin{array}{ll}
1 & 0
\end{array}\right], & D=0, & Q=\left[\begin{array}{ll}
0 & 0
\end{array}\right] .
\end{array}
$$

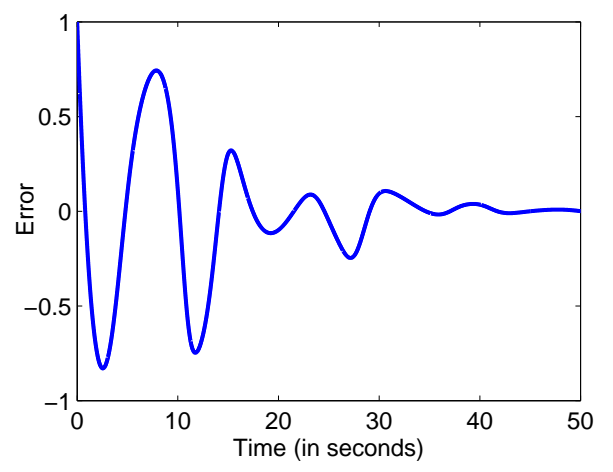

Figure 3. Plot of the error (plant output) for Example 5.1 
It is easy to verify that $A$ is stable and the pair of matrices $\left(\left[\begin{array}{ll}C & Q\end{array}\right],\left[\begin{array}{ll}A & P \\ 0 & S\end{array}\right]\right)$ is detectable. The regulator equations (2.17), (2.18) can be solved, because they reduce to simple algebraic equations. We get

$$
\pi\left(w_{1}, w_{2}\right)=\left[\begin{array}{c}
0 \\
w_{1}
\end{array}\right], \quad \gamma\left(w_{1}, w_{2}\right)=\frac{w_{1}+w_{2}-w_{1}^{4}+\sin \left(w_{1}\right)}{1+w_{1}^{2}} \quad \forall w_{1}, w_{2} \in \mathbb{R} .
$$

Hence all the conditions in Corollary 4.3 hold. The linearization of $\gamma$ at $(0,0)$ is $\Gamma=\left[\begin{array}{ll}2 & 1\end{array}\right]$. The matrix $\mathcal{A}$ in (2.21) is exponentially stable for $C_{c}=\Gamma$ and $B_{c}=\left[\begin{array}{c}-0.2 \\ -0.02\end{array}\right]$. We have now completely determined a second order controller of the form (4.8) which, according to Corollary 4.3, is a controller of minimal order that solves the local error feedback regulator problem for the system under consideration. Note that in this example, the observer-based design outlined above Remark 2.9 would have resulted in a controller of order $n_{c}=6$.

We performed a simulation in MATLAB Simulink using the initial conditions $x_{1}(0)=1, x_{2}(0)=-1, \xi_{1}(0)=0, \xi_{2}(0)=0, w_{1}(0)=0.5$ and $w_{2}(0)=0.25$. The resulting error tends to zero, as seen in Figure 3.

Example 5.2. Consider the second order nonlinear plant

$$
\dot{x}_{1}=x_{2}+x_{1}^{2}-w_{1}^{2}, \quad \dot{x}_{2}=-x_{1}-x_{2}+u
$$

with output $y=x_{1}$. The disturbance signal $w=\left[\begin{array}{c}w_{1} \\ w_{2}\end{array}\right]$ is generated by the exosystem

$$
\left[\begin{array}{l}
\dot{w}_{1} \\
\dot{w}_{2}
\end{array}\right]=\left[\begin{array}{cc}
0 & 1 \\
-1 & 0
\end{array}\right]\left[\begin{array}{l}
w_{1} \\
w_{2}
\end{array}\right] .
$$

The control objective is to ensure that $\lim _{t \rightarrow \infty} y(t)=0$, i.e. the error is $e=x_{1}$. The regulator equations (2.17), (2.18) for the above plant and exosystem reduce to algebraic equations that have the unique solution

$$
\pi(w)=\left[\begin{array}{c}
0 \\
w_{1}^{2}
\end{array}\right], \quad \gamma(w)=w_{1}^{2}+2 w_{1} w_{2} \quad \forall w \in \mathbb{R}^{2} .
$$

In this example, we have

$$
\begin{aligned}
& A=\left[\begin{array}{cc}
0 & 1 \\
-1 & -1
\end{array}\right], \quad B=\left[\begin{array}{l}
0 \\
1
\end{array}\right], \quad P=\left[\begin{array}{ll}
0 & 0 \\
0 & 0
\end{array}\right], \quad S=\left[\begin{array}{cc}
0 & 1 \\
-1 & 0
\end{array}\right], \\
& C=\left[\begin{array}{ll}
1 & 0
\end{array}\right], \quad D=0, \quad Q=\left[\begin{array}{ll}
0 & 0
\end{array}\right] .
\end{aligned}
$$

The exosystem clearly has no influence on the linearized plant or on the error.

We claim that there exists no second order controller of the form (2.9) that solves the local error feedback regulator problem for the above plant and exosystem. Thus a minimal order controller that solves this problem must be of order at least 3 . We will establish the above claim by contradiction. To this end, we assume that the controller (2.9) with order $n_{c}=2$ solves the local error feedback regulator problem. Recall the matrices $F, G$ and $K$ from (2.10) obtained by linearizing the controller. Since the controller (2.9) solves the regulator problem, its closed-loop system with 
the plant is locally exponentially stable, i.e. the matrix $\mathcal{A}_{f}$ in (4.7) is stable. Lemma 2.4 gives that there exist $C^{2}$ maps $\pi: W^{o} \rightarrow \mathbb{R}^{2}$ and $\sigma: W^{o} \rightarrow \mathbb{R}$, where $W^{o} \subset \mathbb{R}^{2}$ is an open set containing zero, such that $\pi(0)=0, \sigma(0)=0$ and (2.12)-(2.14) hold. Since the solution to the regulator equations (2.17), (2.18) for this example is unique and is given by (5.3), comparing (2.12) with (2.17) and (2.14) with (2.18), we get

$$
\pi(w)=\left[\begin{array}{c}
0 \\
w_{1}^{2}
\end{array}\right], \quad \theta(\sigma(w))=w_{1}^{2}+2 w_{1} w_{2} \quad \forall w \in \mathbb{R}^{2} .
$$

Since $\sigma$ is a $C^{2}$ function, using Taylor's theorem [34, Theorem 10, page 179], $\sigma(w)$ and $\frac{\partial \sigma(w)}{\partial w}$ can be expressed as

$$
\begin{aligned}
& \sigma(w)=L\left[\begin{array}{l}
w_{1} \\
w_{2}
\end{array}\right]+\frac{1}{2}\left[\begin{array}{lll}
a_{1} & b_{1} & 2 c_{1} \\
a_{2} & b_{2} & 2 c_{2}
\end{array}\right]\left[\begin{array}{c}
w_{1}^{2} \\
w_{2}^{2} \\
w_{1} w_{2}
\end{array}\right]+\left[\begin{array}{l}
r_{1}(w) \\
r_{2}(w)
\end{array}\right], \\
& \frac{\partial \sigma(w)}{\partial w}=L+\left[\begin{array}{ll}
a_{1} w_{1}+c_{1} w_{2} & b_{1} w_{2}+c_{1} w_{1} \\
a_{2} w_{1}+c_{2} w_{2} & b_{2} w_{2}+c_{2} w_{1}
\end{array}\right]+\left[\begin{array}{l}
r_{3}(w) \\
r_{4}(w)
\end{array}\right],
\end{aligned}
$$

where $L$ is a real $2 \times 2$ matrix, $a_{l}, b_{l}, c_{l} \in \mathbb{R}$ for $l=1,2, r_{1}$ and $r_{2}$ are real-valued $C^{2}$ functions satisfying $\left(\left|r_{1}(w)\right|+\left|r_{2}(w)\right|\right) /\|w\|^{2} \rightarrow 0$ as $\|w\| \rightarrow 0$ and $r_{3}$ and $r_{4}$ are real-valued $C^{1}$ functions satisfying $\left(\left|r_{3}(w)\right|+\left|r_{4}(w)\right|\right) /\|w\| \rightarrow 0$ as $\|w\| \rightarrow 0$. Similarly $\theta(w)$ can be expressed as

$$
\theta(w)=K w+R(w),
$$

where $R$ is a real-valued $C^{2}$ function satisfying $|R(w)| /\|w\| \rightarrow 0$ as $\|w\| \rightarrow 0$.

Next we show that $L=0$. Linearizing (2.13) around the origin gives $L S=$ $F L$. Since $F$ is a real matrix, either $\sigma_{p}(S) \cap \sigma_{p}(F)=\emptyset$ or $\sigma_{p}(S)=\sigma_{p}(F)$. If $\sigma_{p}(S) \cap \sigma_{p}(F)=\emptyset$, then $L S=F L$ easily implies that $L=0$. So suppose that $\sigma_{p}(S)=\sigma_{p}(F)$. Then $F=T^{-1} S T$ for some invertible $2 \times 2$ matrix $T$ and the exponential stability of $\mathcal{A}_{f}$ implies that $K \neq 0$. From $L S=F L$ and $F=T^{-1} S T$ we get $T L S=S T L$, which implies that $T L=\left[\begin{array}{cc}\alpha & -\beta \\ \beta & \alpha\end{array}\right]$ for some $\alpha, \beta \in \mathbb{C}$. So either $L=0$ or $L$ is invertible. From the second equation in (5.4) we get that

$$
\left[\frac{\partial \theta(\sigma(w))}{\partial w}\right]_{w=0}=K L=\left[\begin{array}{ll}
0 & 0
\end{array}\right]
$$

which, along with $K \neq 0$, gives that $L=0$.

Substituting for $\sigma(w)$ and $\frac{\partial \sigma(w)}{\partial w}$ from (5.5) and (5.6) into (2.13) and equating the quadratic terms in the resulting expression (while using $L=0$ ) gives

$$
\left[\begin{array}{cc}
a_{1} w_{1}+c_{1} w_{2} & b_{1} w_{2}+c_{1} w_{1} \\
a_{2} w_{1}+c_{2} w_{2} & b_{2} w_{2}+c_{2} w_{1}
\end{array}\right] S\left[\begin{array}{l}
w_{1} \\
w_{2}
\end{array}\right]=\frac{1}{2} F\left[\begin{array}{lll}
a_{1} & b_{1} & 2 c_{1} \\
a_{2} & b_{2} & 2 c_{2}
\end{array}\right]\left[\begin{array}{c}
w_{1}^{2} \\
w_{2}^{2} \\
w_{1} w_{2}
\end{array}\right] .
$$

By comparing the coefficients of $w_{1}^{2}, w_{2}^{2}$ and $w_{1} w_{2}$ on both sides of the above equation we get, after a simple calculation, that

$$
F\left[\begin{array}{l}
c_{1} \\
c_{2}
\end{array}\right]=\left[\begin{array}{l}
a_{1}-b_{1} \\
a_{2}-b_{2}
\end{array}\right], \quad F^{2}\left[\begin{array}{l}
c_{1} \\
c_{2}
\end{array}\right]=-4\left[\begin{array}{l}
c_{1} \\
c_{2}
\end{array}\right] .
$$


The second equation in (5.9) means that either $\left[\begin{array}{c}c_{1} \\ c_{2}\end{array}\right]=0$ or $F$ has eigenvalues at $\pm 2 i$. We will prove below that neither of these possibilities can occur. This means that there is no $C^{2}$ map $\sigma$ for which (2.13) holds for any second order controller. This contradicts Lemma 2.4 and hence no second order controller of the form (2.9) can solve the local error feedback regulator problem.

Suppose that $\left[\begin{array}{l}c_{1} \\ c_{2}\end{array}\right]=0$. Then the first equation in (5.9) gives $\left[\begin{array}{l}a_{1} \\ a_{2}\end{array}\right]=\left[\begin{array}{l}b_{1} \\ b_{2}\end{array}\right]$. Using this, (5.4), (5.5), (5.7) and $L=0$ we get the following contradiction:

$$
\begin{aligned}
& 1=\lim _{w_{1} \rightarrow 0} \frac{\theta\left(\sigma\left(w_{1}, 0\right)\right)}{w_{1}^{2}}=\lim _{w_{1} \rightarrow 0} \frac{K \sigma\left(w_{1}, 0\right)+R\left(\sigma\left(w_{1}, 0\right)\right)}{w_{1}^{2}}=K\left[\begin{array}{l}
a_{1} \\
a_{2}
\end{array}\right], \\
& 0=\lim _{w_{2} \rightarrow 0} \frac{\theta\left(\sigma\left(0, w_{2}\right)\right)}{w_{2}^{2}}=\lim _{w_{2} \rightarrow 0} \frac{K \sigma\left(0, w_{2}\right)+R\left(\sigma\left(0, w_{2}\right)\right)}{w_{2}^{2}}=K\left[\begin{array}{l}
a_{1} \\
a_{2}
\end{array}\right] .
\end{aligned}
$$

Here we have used the following fact: Denote $w_{\alpha}=\left[\alpha w_{1},(1-\alpha) w_{2}\right]$ and $\|a\|=$ $\sqrt{a_{1}^{2}+a_{2}^{2}}$. Then, since $\left[\begin{array}{c}c_{1} \\ c_{2}\end{array}\right]=0$ and $\left[\begin{array}{l}a_{1} \\ a_{2}\end{array}\right]=\left[\begin{array}{l}b_{1} \\ b_{2}\end{array}\right]$, for $\alpha=0$ and $\alpha=1$

$$
\lim _{w_{\alpha} \rightarrow 0} \frac{\left|R\left(\sigma\left(w_{\alpha}\right)\right)\right|}{\left\|w_{\alpha}\right\|^{2}}=\lim _{w_{\alpha} \rightarrow 0} \frac{\left|R\left(\sigma\left(w_{\alpha}\right)\right)\right|}{\left\|\sigma\left(w_{\alpha}\right)\right\|} \frac{\left\|\sigma\left(w_{\alpha}\right)\right\|}{\left\|w_{\alpha}\right\|^{2}}=\|a\| \lim _{w_{\alpha} \rightarrow 0} \frac{\left|R\left(\sigma\left(w_{\alpha}\right)\right)\right|}{\left\|\sigma\left(w_{\alpha}\right)\right\|}=0 .
$$

Suppose that $F$ has eigenvalues at $\pm 2 i$, i.e. $F=T^{-1}\left[\begin{array}{cc}0 & 2 \\ -2 & 0\end{array}\right] T$ for some invertible real matrix $T$. We assume (without loss of generality) that the coordinate system of the controller has been chosen so that $F=\left[\begin{array}{cc}0 & 2 \\ -2 & 0\end{array}\right]$. Substituting this $F$ into (5.8) and comparing the coefficients of $w_{1}^{2}, w_{2}^{2}$ and $w_{1} w_{2}$ on both sides of the resulting equation gives, after a simple calculation, that $\left[\begin{array}{l}a_{1} \\ a_{2}\end{array}\right]=\left[\begin{array}{c}c_{2} \\ -c_{1}\end{array}\right]$ and $\left[\begin{array}{l}b_{1} \\ b_{2}\end{array}\right]=\left[\begin{array}{c}-c_{2} \\ c_{1}\end{array}\right]$. Using this, (5.4), (5.5), (5.7), (5.10) and $L=0$ we get the following contradiction:

$$
\begin{gathered}
1=\lim _{w_{1} \rightarrow 0} \frac{\theta\left(\sigma\left(w_{1}, 0\right)\right)}{w_{1}^{2}}=\lim _{w_{1} \rightarrow 0} \frac{K \sigma\left(w_{1}, 0\right)+R\left(\sigma\left(w_{1}, 0\right)\right)}{w_{1}^{2}}=K\left[\begin{array}{c}
c_{2} \\
-c_{1}
\end{array}\right], \\
0=\lim _{w_{2} \rightarrow 0} \frac{\theta\left(\sigma\left(0, w_{2}\right)\right)}{w_{2}^{2}}=\lim _{w_{2} \rightarrow 0} \frac{K \sigma\left(0, w_{2}\right)+R\left(\sigma\left(0, w_{2}\right)\right)}{w_{2}^{2}}=-K\left[\begin{array}{c}
c_{2} \\
-c_{1}
\end{array}\right] .
\end{gathered}
$$

Although no second order controller can solve the local error feedback regulator problem for the plant and the exosystem in this example, it is easy to find a third order controller that does solve this problem. Define the matrices $\Phi$ and $\Lambda$ and the $\operatorname{map} \tau: \mathbb{R}^{2} \rightarrow \mathbb{R}^{3}$ as follows:

$$
\Phi=\left[\begin{array}{ccc}
0 & 0 & 0 \\
0 & 0 & -2 \\
0 & 2 & 0
\end{array}\right], \quad \Lambda=\left[\begin{array}{lll}
0.5 & 1 & 0.5
\end{array}\right], \quad \tau\left(w_{1}, w_{2}\right)=\left[\begin{array}{c}
w_{1}^{2}+w_{2}^{2} \\
2 w_{1} w_{2} \\
w_{1}^{2}-w_{2}^{2}
\end{array}\right] .
$$

Using Definition 2.7, it can be verified that the autonomous system $\left(\mathbb{R}^{2}, S, \gamma\right)$ is immersed into the autonomous system $\left(\mathbb{R}^{3}, \Phi, \Lambda\right)$ via the mapping $\tau$. Clearly $(\Lambda, \Phi)$ is detectable, $A$ is stable, $\mathbf{G}(0) \neq 0$ and $\mathbf{G}( \pm 2 i) \neq 0$. Hence all the conditions of Theorem 4.1 are satisfied and a third order controller of the form (4.2) with $\phi(\xi)=\Phi \xi$ and $\lambda(\xi)=\Lambda \xi$ that solves the regulator problem can be constructed. In contrast, the observer-based controller design outlined above Remark 2.9 would result in a controller of order $n_{c}=8$. 
Example 5.3. Consider the boost converter shown in Figure 1, for which we already gave some background in Section 1. We assume that the switches are operated so fast that instantaneous values of the currents and voltages in the switches can be replaced by their average values over short time intervals, for instance, the sampling period, or the time interval between two consecutive turn-on times of the upper switch (which need not be of constant length). $q$ and $\bar{q}$ are complementary binary signals and $q=1$ means that the upper switch is closed. We denote by $\mathcal{D}$ the short-time average value of $q$, so that $\mathcal{D} \in[0,1]$. The state variables $z_{1}, z_{2}$ and the inputs $v$ and $i_{e}$ are considered practically equal to their short-time averaged values. It is easy to derive the equations corresponding to the averaged variables:

$$
C \dot{z}_{1}=-i_{e}-\frac{z_{1}}{R}+\mathcal{D} z_{2}, \quad L \dot{z}_{2}=-r z_{2}+v-\mathcal{D} z_{1},
$$

where $C>0$ is the capacitance, $L>0$ is the inductance, $R>0$ is the resistance of the load and $r>0$ is a small resistance that accounts for the losses in the inductor. For a rigorous justification of this averaging process we refer to [28], [32], 333].

To bring these equations into the framework of Section 2, we consider an operating point (an equilibrium state) corresponding to the inputs $v=v_{0}>0, i_{e}=0, \mathcal{D}=$ $\mathcal{D}_{0} \in(0,1)$. The corresponding equilibrium state $\left[\begin{array}{ll}z_{10} & z_{20}\end{array}\right]^{\top}$ can be computed by setting $\dot{z}_{1}=0$ and $\dot{z}_{2}=0$ in (5.11):

$$
-\frac{z_{10}}{R}+\mathcal{D}_{0} z_{20}=0, \quad-r z_{20}+v_{0}-\mathcal{D}_{0} z_{10}=0,
$$

whence

$$
z_{10}=\frac{\mathcal{D}_{0}}{\frac{r}{R}+\mathcal{D}_{0}^{2}} v_{0}, \quad z_{20}=\frac{\frac{1}{R}}{\frac{r}{R}+\mathcal{D}_{0}^{2}} v_{0} .
$$

It is assumed that this is a desirable equilibrium point, i.e. $z_{10}$ is exactly the reference output voltage. The input voltage $v$ may deviate from $v_{0}$ (for instance, batteries running on low charge), but this deviation is considered to be a constant at the time scale of interest. The disturbance current $i_{e}$ is considered to be sinusoidal, with known frequency $\alpha>0$. For instance, this disturbance might be caused by a singlephase $\mathrm{DC} / \mathrm{AC}$ converter taking its power from this point and delivering current to some AC load, in which case $\alpha$ would be twice the frequency of the output voltage. Such a configuration would be typical for an uninterruptible power supply (UPS). The state and input variables will be the deviations of the original variables from their values at the operating point:

$$
x_{1}=z_{1}-z_{10}, \quad x_{2}=z_{2}-z_{20}, \quad w_{1}=v-v_{0}, \quad u=\mathcal{D}-\mathcal{D}_{0} .
$$

Using (5.11), and the notation $w_{2}=i_{e}$, the deviations satisfy the equations:

$$
\begin{aligned}
& \dot{x}_{1}=-\frac{x_{1}}{R C}+\frac{\mathcal{D}_{0}+u}{C} x_{2}+\frac{z_{20}}{C} u-\frac{1}{C} w_{2}, \\
& \dot{x}_{2}=-\frac{\mathcal{D}_{0}+u}{L} x_{1}-\frac{r}{L} x_{2}-\frac{z_{10}}{L} u+\frac{1}{L} w_{1} .
\end{aligned}
$$

The disturbance signal $w_{1}$ is an unknown constant while $w_{2}(t)=a \cos (\alpha t+\phi)$, where $a$ and $\phi$ are unknown. We assume that $w_{1}$ and $w_{2}$ are generated by the exosystem

$$
\dot{w}=S w, \quad w=\left[\begin{array}{c}
w_{1} \\
w_{2} \\
w_{3}
\end{array}\right], \quad S=\left[\begin{array}{ccc}
0 & 0 & 0 \\
0 & 0 & \alpha \\
0 & -\alpha & 0
\end{array}\right] .
$$


We want to regulate $x_{1}$ to zero and therefore the error is

$$
e=x_{1} \text {. }
$$

It is well known that it is difficult to control the higher voltage in a boost converter because of the unstable zero dynamics (which can be seen from the presence of a right-half plane zero in the transfer function of the linearization from $u$ to $x_{1}$ ), see for instance [35] and the references therein.

Denote $x=\left[\begin{array}{l}x_{1} \\ x_{2}\end{array}\right]$. We can rewrite (5.12), (5.13) and (5.15) in the standard form of (2.1) and (2.4), by defining the appropriate $C^{\infty}$ functions $f$ and $h$ on $\mathbb{R}^{2} \times \mathbb{R} \times \mathbb{R}^{3}$. These functions satisfy $f(0,0,0)=0$ and $h(0,0,0)=0$. The linearization of (5.12), (5.13) and (5.15) around $(0,0,0)$ is as in (2.7)-(2.8), with

$$
\begin{array}{ccc}
A=\left[\begin{array}{cc}
-\frac{1}{R C} & \frac{\mathcal{D}_{0}}{C} \\
-\frac{\mathcal{D}_{0}}{L} & -\frac{r}{L}
\end{array}\right], & B=\left[\begin{array}{c}
\frac{z_{20}}{C} \\
-\frac{z_{10}}{L}
\end{array}\right], & P=\left[\begin{array}{ccc}
0 & -\frac{1}{C} & 0 \\
\frac{1}{L} & 0 & 0
\end{array}\right], \\
C=\left[\begin{array}{ll}
1 & 0
\end{array}\right], & D=0, & Q=\left[\begin{array}{lll}
0 & 0 & 0
\end{array}\right] .
\end{array}
$$

It is easy to see (from $\operatorname{trace} A<0$ and $\operatorname{det} A>0$ ) that $A$ is stable. The detectability assumption contained in Corollary 4.3 can be verified (for any combination of parameter values) using the Hautus test.

The nonlinear regulator equations (2.17), with the notation $\pi=\left[\begin{array}{l}\pi^{1} \\ \pi^{2}\end{array}\right]$, are

$$
\begin{aligned}
& \alpha \frac{\partial \pi^{1}}{\partial w_{2}} w_{3}-\alpha \frac{\partial \pi^{1}}{\partial w_{3}} w_{2}=-\frac{\pi^{1}}{R C}+\frac{\mathcal{D}_{0}+\gamma}{C} \pi^{2}+\frac{z_{20}}{C} \gamma-\frac{1}{C} w_{2}, \\
& \alpha \frac{\partial \pi^{2}}{\partial w_{2}} w_{3}-\alpha \frac{\partial \pi^{2}}{\partial w_{3}} w_{2}=-\frac{\mathcal{D}_{0}+\gamma}{L} \pi^{1}-\frac{r}{L} \pi^{2}-\frac{z_{10}}{L} \gamma+\frac{1}{L} w_{1} .
\end{aligned}
$$

The regulator equation (2.18) is $\pi^{1}=0$. Using this, we rewrite the above as

$$
\begin{gathered}
0=\frac{\mathcal{D}_{0}+\gamma}{C} \pi^{2}+\frac{z_{20}}{C} \gamma-\frac{1}{C} w_{2}, \\
\alpha \frac{\partial \pi^{2}}{\partial w_{2}} w_{3}-\alpha \frac{\partial \pi^{2}}{\partial w_{3}} w_{2}=-\frac{r}{L} \pi^{2}-\frac{z_{10}}{L} \gamma+\frac{1}{L} w_{1} .
\end{gathered}
$$

Substituting $\gamma$ from (5.17) into (5.16) we get

$$
\begin{aligned}
& \left(\mathcal{D}_{0}+\frac{L}{z_{10}}\left[-\alpha \frac{\partial \pi^{2}}{\partial w_{2}} w_{3}+\alpha \frac{\partial \pi^{2}}{\partial w_{3}} w_{2}-\frac{r}{L} \pi^{2}+\frac{1}{L} w_{1}\right]\right) \pi^{2} \\
& +z_{20} \frac{L}{z_{10}}\left[-\alpha \frac{\partial \pi^{2}}{\partial w_{2}} w_{3}+\alpha \frac{\partial \pi^{2}}{\partial w_{3}} w_{2}-\frac{r}{L} \pi^{2}+\frac{1}{L} w_{1}\right]-w_{2}=0 .
\end{aligned}
$$

This is a first order quasilinear PDE in the unknown function $\pi^{2}: \mathbb{R}^{3} \rightarrow \mathbb{R}$, with no boundary conditions, only a one-point condition: $\pi^{2}(0)=0$. We will solve it in a neighborhood of $0 \in \mathbb{R}^{3}$. Our approach is to determine $\pi^{2}$ on circles of the following type: $w_{1}$ is constant, $w_{2}=\rho \cos \tau$ and $w_{3}=\rho \sin \tau$, where $\rho>0$ is a constant and $\tau \in[0,2 \pi)$. The motivation for our approach is that on such circles, the PDE (5.18) becomes an ODE. (In fact, these circles are the projections of the characteristic 
curves of (5.18) in $\mathbb{R}^{4}$, onto the space $\mathbb{R}^{3}$ with coordinates $w_{1}, w_{2}$ and $w_{3}$.) For each fixed $w_{1}$ and $\rho$, we define a function $\psi$ on $[0,2 \pi)$ by

$$
\psi(\tau)=\pi^{2}\left(w_{1}, \rho \cos \tau, \rho \sin \tau\right) .
$$

It follows by the chain rule that

$$
\frac{\mathrm{d} \psi}{\mathrm{d} \tau}=-\frac{\partial \pi^{2}}{\partial w_{2}} \rho \sin \tau+\frac{\partial \pi^{2}}{\partial w_{3}} \rho \cos \tau=-\frac{\partial \pi^{2}}{\partial w_{2}} w_{3}+\frac{\partial \pi^{2}}{\partial w_{3}} w_{2} .
$$

Using the previous expression, (5.18) can be rewritten as

$$
\frac{\mathrm{d} \psi}{\mathrm{d} \tau}=\frac{r \psi^{2}+\left(r z_{20}-w_{1}-\mathcal{D}_{0} z_{10}\right) \psi-z_{20} w_{1}+z_{10} \rho \cos \tau}{\alpha L\left(\psi+z_{20}\right)} .
$$

This ODE has to be solved for $\psi$ that satisfies the periodic boundary condition $\psi(0)=\psi(2 \pi)$. From the function $\psi$, corresponding to different values of $w_{1}$ and $\rho$, we will then construct the function $\pi^{2}$ using (5.19).

First we claim that if $\rho<\mathcal{D}_{0} z_{20}$ and $\psi(0)>-z_{20}$, then there exists a global in time unique solution to (5.20). Our claim is a consequence of the following two facts: (i) If the two inequalities mentioned above hold, then $\psi(\tau)>-z_{20}$ for all $\tau \geq 0$ along the trajectory of (5.20). Indeed, if this were false, then for some $t>0$ $\lim _{\tau \rightarrow t} \psi(\tau)=-z_{20}$. Let $t$ be the smallest such number, so that $\psi(\tau)>-z_{20}$ for $\tau<t$. But such a $t$ cannot exist since the limit of $\frac{\mathrm{d} \psi}{\mathrm{d} \tau}$ (as given in (5.20) ) for $\psi \rightarrow-z_{20}, \psi>-z_{20}$ is $+\infty$. (ii) When $\psi$ is large and positive, the right side of (5.20) behaves linearly in $\psi$, so that $\psi$ cannot escape to $+\infty$ in finite time.

In the sequel, we assume that

$$
\rho<\beta \mathcal{D}_{0} z_{20} \quad \text { for some } \quad 0<\beta<1, \quad \psi(0)>-z_{20} .
$$

We will solve (5.20) with the periodic boundary condition under the assumptions (5.21) and $\mathcal{D}_{0} z_{10}>r z_{20}$. The latter assumption, which is realistic since $r$ is usually very small, is needed because of the following fact:

Claim. If $\mathcal{D}_{0} z_{10}=r z_{20}$, then there is no local solution $\pi^{2}$ to (5.18) which is of class $C^{2}$ and satisfies $\pi^{2}(0)=0$.

Indeed, if our claim is false and such a $\pi^{2}$ exists, then for all values of $w_{1}$ and $\rho$ sufficiently small there exists a solution $\psi$ to (5.20) that satisfies $\psi(0)=\psi(2 \pi)$, obtained from $\pi^{2}$ via (5.19). Clearly there exists a $w_{1}<0$ and $\rho>0$ such that $\psi$, as defined above, satisfies $\left(-\psi(0)-z_{20}\right) w_{1}>\rho$. But for this $\psi$, since $\mathcal{D}_{0} z_{10}=r z_{20}$ (by assumption), it is easy to verify that the right side of (5.20) will be positive for all $\tau \in[0,2 \pi)$. This gives us the contradiction $\psi(0)<\psi(2 \pi)$, which proves our claim.

We will next identify the range of values for $w_{1}$ and $\rho$ for which (5.20) has a solution satisfying the periodic boundary condition. By solving the quadratic equation obtained from (5.20) by setting $\frac{\mathrm{d} \psi}{\mathrm{d} \tau}=0$ and choosing $\cos \tau= \pm 1$, we define for each $w_{1}$ and $\rho$ the scalars $\psi_{1}$ and $\psi_{2}$ as follows:

$$
\psi_{1}=\frac{-\left(r z_{20}-w_{1}-\mathcal{D}_{0} z_{10}\right)-\sqrt{\left(r z_{20}-w_{1}-\mathcal{D}_{0} z_{10}\right)^{2}-4 r\left(-z_{20} w_{1}+z_{10} \rho\right)}}{2 r},
$$




$$
\psi_{2}=\frac{-\left(r z_{20}-w_{1}-\mathcal{D}_{0} z_{10}\right)-\sqrt{\left(r z_{20}-w_{1}-\mathcal{D}_{0} z_{10}\right)^{2}-4 r\left(-z_{20} w_{1}-z_{10} \rho\right)}}{2 r} .
$$

Let $w_{1}^{\max }=\mathcal{D}_{0} z_{10}-r z_{20}$. For each $w_{1}$ satisfying $\left|w_{1}\right|<w_{1}^{\max }$ let

$$
\rho_{w_{1}}^{\max }=\min \left\{\beta \mathcal{D}_{0} z_{20}, \frac{\left(r z_{20}-w_{1}-\mathcal{D}_{0} z_{10}\right)^{2}}{4 r z_{10}}-\frac{z_{20}\left|w_{1}\right|}{z_{10}}\right\} .
$$

Consider the set $\mathcal{W} \subset \mathbb{R}^{2}$ defined by $\mathcal{W}=\left\{\left(w_{1}, \rho\right)|| w_{1} \mid<w_{1}^{\max }, 0 \leq \rho<\rho_{w_{1}}^{\max }\right\}$. For each $\left(w_{1}, \rho\right) \in \mathcal{W}$ and every sufficiently small $\varepsilon>0$ the following hold:

(1) $\psi_{1}, \psi_{2} \in \mathbb{R}$ with $\psi_{1}>\psi_{2}>-z_{20}$ and as $w_{1}$ and $\rho$ tend to zero, $\psi_{1}$ and $\psi_{2}$ tend to zero,

(2) the solution $\psi$ of (5.20) with $\psi(0)=\psi_{1}+\varepsilon$ satisfies $\psi(2 \pi)<\psi(0)$,

(3) the solution $\psi$ of (5.20) with $\psi(0)=\psi_{2}-\varepsilon$ satisfies $\psi(2 \pi)>\psi(0)$.

It is easy to verify item (1) via algebraic manipulations. To see that items (2) and (3) hold, observe that when $\psi$ is near but larger than $\psi_{1}$ the right side of (5.20) is negative and when it is near but smaller than $\psi_{2}$ the right side of (5.20) is positive. From items (1)-(3) it follows that there exists a $\psi_{0} \in\left[\psi_{2}, \psi_{1}\right]$ such that the solution of (5.20) with $\psi(0)=\psi_{0}$ satisfies $\psi(0)=\psi(2 \pi)$. A simple algorithm for estimating $\psi_{0}$, which uses the fact that no two trajectories of (5.20) can intersect in the $\psi-\tau$ plane, is as follows. For $n \geq 1$, let $\psi^{n}$ denote the solution of (5.20) with $\psi^{1}(0)=\left(\psi_{1}+\psi_{2}\right) / 2$ and $\psi^{n}(0)=\psi^{n-1}(2 \pi)$ for $n>1$. It follows from items (1)-(3) that $\psi^{n}(0)>-z_{20}$ for all $n$ and $\psi^{n}(0) \rightarrow \psi_{0}$ as $n \rightarrow \infty$.

For each $\left(w_{1}, \rho\right) \in \mathcal{W}$, we first compute $\psi_{0}$ and the solution $\psi$ to (5.20) that satisfies $\psi(0)=\psi(2 \pi)=\psi_{0}$. Then, using (5.19), we define the function $\pi^{2}$ on the domain $\left\{\left(w_{1}, w_{2}, w_{3}\right)|| w_{1} \mid<w_{1}^{\max }, 0 \leq \sqrt{w_{2}^{2}+w_{3}^{2}}<\rho_{w_{1}}^{\max }\right\}$. From the center manifold theorem [20, Theorem 3.22], there exists a smooth locally attractive invariant manifold for the dynamics of (5.20) (with $w_{2}$ in place of $\rho \cos \tau$ ) and (5.14). Since $\pi^{2}$ is constructed using periodic solutions to (5.20) and (5.14), which lie on the above manifold for small initial data, we can conclude that it defines a manifold which coincides with the above manifold locally. Hence $\pi^{2}$ is locally smooth. The function $\gamma$ can be obtained using (5.16) and it will also be locally smooth.

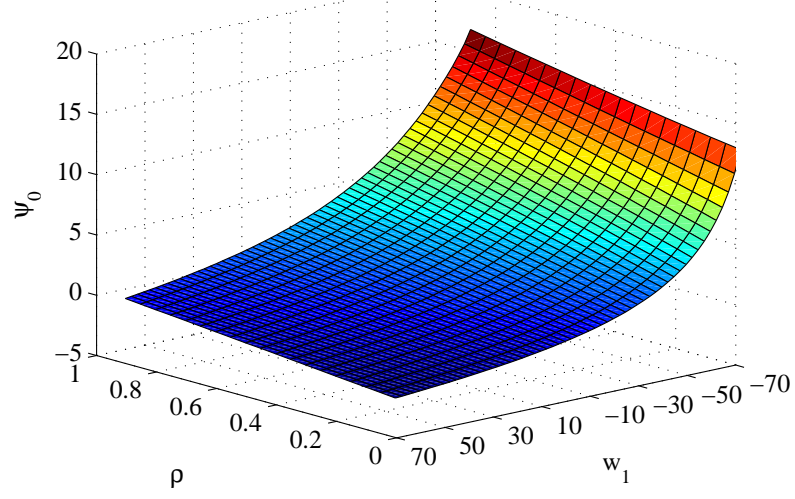

Figure 4. Surface plot of the initial conditions $\psi_{0}$ (as a function of $w_{1}$ and $\rho$ ) for which the solution $\psi$ to (5.20) satisfies $\psi_{0}=\psi(0)=\psi(2 \pi)$. 
For our simulation we choose the nominal input voltage $v_{0}=100 \mathrm{~V}$, the desired voltage (across the load) $z_{10}=400 \mathrm{~V}$, the load resistance $R=400 \Omega, r=0.25 \Omega$ and $\beta=0.9$ ( $\beta$ appears in (5.21) ). Assuming that the switching frequency of the switches in Figure 1 is $20 \mathrm{KHz}$, we choose $L=4 \mathrm{mH}$ and $C=40 \mu \mathrm{F}$ to reduce the ripple in the inductor current and the voltage across the load to reasonable values. The disturbance frequency is $\alpha=200 \pi \mathrm{rad} / \mathrm{sec}$ (twice the nominal grid frequency). From the above values we get that $\mathcal{D}_{0}=0.2474$ and $z_{20}=4.04 \mathrm{~A}$. We first identify the set $\mathcal{W}$ described earlier and compute $\psi_{0}$ for each $\left(w_{1}, \rho\right) \in \mathcal{W}$. Figure 4 shows the surface plot of $\psi_{0}$. As expected from the local smoothness of $\pi^{2}$, the surface of $\psi_{0}$ is smooth near $\left(w_{1}, \rho\right)=(0,0)$. It is clear from the plot that $\psi_{0}$ is a smooth function on $\mathcal{W}$. From the continuous dependence of solutions to (5.20) on initial conditions, we can conclude that $\pi^{2}$ and $\gamma$ (computed as discussed earlier) will be smooth on their common domain of definition. In Figure 5, we plot $\psi$ and $\gamma$ on the circles contained in the set $\left\{\left(w_{1}, w_{2}, w_{3}\right) \mid w_{1} \in\{-50,0,50\}, \sqrt{w_{2}^{2}+w_{3}^{2}}=0.3\right\}$.

By solving (4.9) we get $\Gamma=\left[\begin{array}{lll}2.53 \times 10^{-3} & 1.06 \times 10^{-4}-2.56 \times 10^{-2}\end{array}\right]$. The ma$\operatorname{trix} \mathcal{A}$ in (2.21) is exponentially stable for $C_{c}=\Gamma$ and $B_{c}=\left[\begin{array}{lll}7.5 & -0.29 & 0.06\end{array}\right]^{\mathrm{T}}$. We have now completely determined a third order controller of the form (4.8) which, according to Corollary 4.3, is a controller of minimal order that solves the local error feedback regulator problem for the system (5.12)-(5.15). We have performed a simulation in Simulink with the following initial conditions: $x_{1}(0)=5, x_{2}(0)=0$, $\xi_{1}(0)=0, \xi_{2}(0)=0, w_{1}(0)=10, w_{2}(0)=0.8$ and $w_{3}(0)=0$. The resulting error decays asymptotically to zero, as seen in Figure 6. We remark that in this example the observer-based design outlined above Remark 2.9 would have resulted in a controller of order $n_{c}=8$.

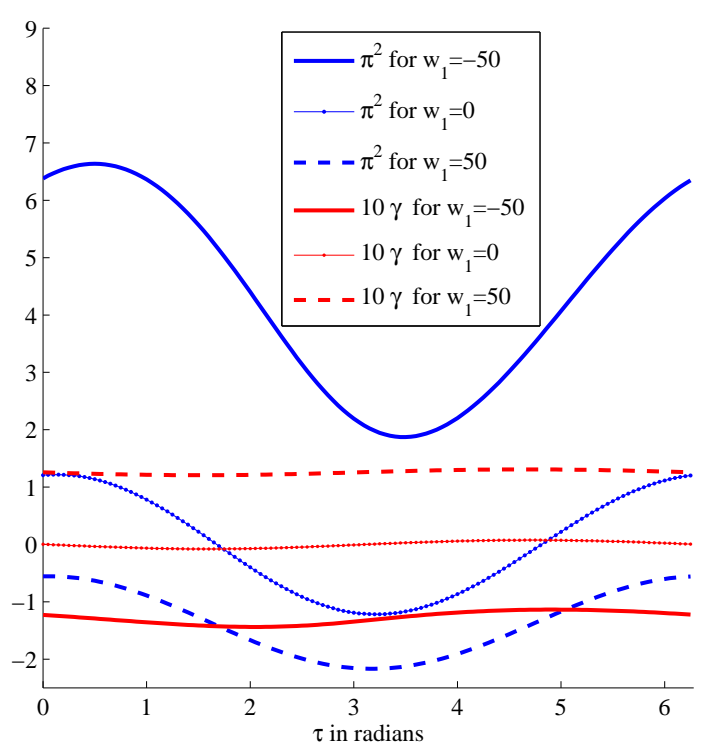

Figure 5. Plot of $\pi^{2}$ and (10 times) $\gamma$ on circles of radius $\rho=0.3$ and $w_{1} \in\{-50,0,50\}$, as a function of the angle $\tau$. All the functions resemble sinusoids. 


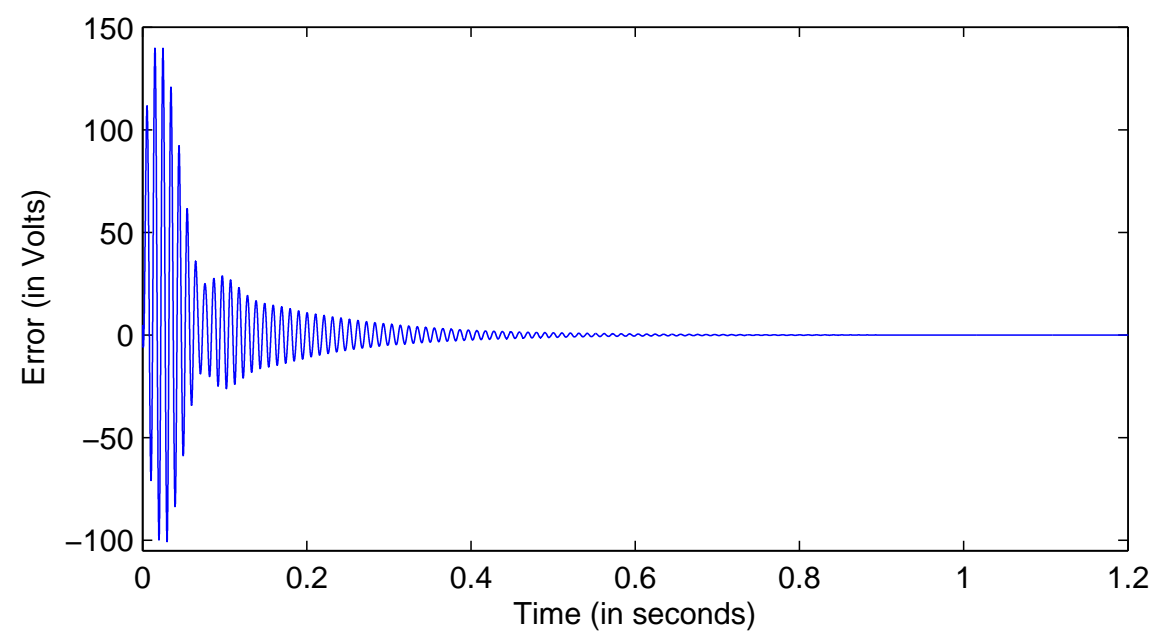

Figure 6 . The error, the deviation of $z_{1}$ from its reference value $(400 \mathrm{~V})$

\section{Conclusions}

In this work we have focussed on finding lower order controllers that solve the local error feedback regulator problem for locally exponentially stable nonlinear plants that are SISO from control input to output. Under certain assumptions, we have presented a methodology for constructing controllers whose order is equal to the order of a detectable immersion associated with the exosystem and a solution to the regulator equations. Future work will address the issue of finding minimal order controllers for multi-input multi-output plants. A relevant research area is the construction of finite-dimensional controllers for regulating the output of nonlinear infinite-dimensional systems (preliminary results are in [36]).

\section{References}

[1] Aguilar, C.O., \& Krener, A.J. (2013). Patchy solution of a Francis-Byrnes-Isidori partial differential equation. Int. J. Robust and Nonlinear Control, 23, 1046-1061.

[2] Anderson, B.D.O., \& Gevers, M.R. (1981). On multivariable pole-zero cancellations and the stability of feedback systems. IEEE Trans. Circuits ES Systems, 28, 830-833.

[3] Astolfi, D., Isidori, A., Marconi, L., \& Praly, L. (2013). Nonlinear output regulation by post-processing internal model for multi-input multi-output systems. Proc. of $9^{\text {th }}$ IFAC Symposium on Nonlinear Control Systems, Toulouse, France (pp. 295-300).

[4] Astolfi, A., \& Ortega, R. (2003). Immersion and invariance: A new tool for stabilization and adaptive control of nonlinear systems. IEEE Trans. Automatic Control, 48, 590-606.

[5] Aubin, J.P., \& Da Prato, G. (1992). Contingent solutions to the center manifold equation. Annales de l'Inst. Henry Poincaré, 9, 13-28. 
[6] Byrnes, C.I., \& Gilliam, D.S. (2007). Approximate solutions of the regulator equations for nonlinear DPS. Proc. of the $46^{\text {th }}$ IEEE Conf. on Decision and Control, New Orleans, LA (pp. 854-859).

[7] Byrnes, C.I., Priscoli, F.D., \& Isidori, A. (1997). Output regulation of uncertain nonlinear systems. Boston: Birkhäuser.

[8] Byrnes, C.I., Priscoli, F.D., Isidori, A., \& Kang, W. (1997). Structurally stable output regulation of nonlinear systems. Automatica, 33, 369-385.

[9] Cao, Y-Y., Lam, J., \& Sun, Y-X. (1998). Static output feedback stabilization: An ILMI approach. Automatica, 34, 1641-1645.

[10] Carr, J. (1981). Applications of Center Manifold Theory. New York: Springer-Verlag.

[11] Chen, Z., \& Huang, J. (2005). Global robust output regulation for output feedback systems. IEEE Trans. Automatic Control, 50, 117-121.

[12] Davison, E.J. (1976). Multivariable tuning regulators: the feedforward and robust control of a general servomechanism problem. IEEE Trans. Aut. Control, 21, 35-47.

[13] Davison, E.J., \& Goldenberg, A. (1975). The robust control of a general servomechanism problem: the servo compensator. Automatica, 11, 461-471.

[14] Desoer, C.A., \& Wang, Y.T. (1978). On the minimum order of a robust servocompensator. IEEE Trans. Automatic Control, 23, 70-73.

[15] Francis, B.A. (1977). The linear multivariable regulator problem. SIAM J. Control and Optimization, 15, 486-505.

[16] Francis, B.A., \& Wonham, W.M. (1975). The internal model principle for linear multivariable regulators. Appl. Math. Optimization, 2, 170-194.

[17] Ghaoui, L.E., Oustry, F., \& AitRami, M. (1997). A cone complementarity linearization algorithm for static output-feedback and related problems. IEEE Trans. Automatic Control, 42, 1171-1176.

[18] Guckenheimer, J., \& Holmes, P. (1983). Nonlinear Oscillations, Dynamical Systems, and Bifurcations of Vector Fields. Applied Mathematical Sciences 42, New York: Springer-Verlag.

[19] Hämäläinen, T., \& Pohjolainen, S. (2000). A finite-dimensional robust controller for system in the CD-algebra. IEEE Trans. Automatic Control, 45, 421-431.

[20] Haragus, M., \& Iooss, G. (2011). Local Bifurcations, Center Manifolds, and Normal Forms in Infinite-Dimensionals Dynamical Systems. Les Ulis: EDP Sciences and London: Springer.

[21] Huang, J. (2004). Nonlinear Output Regulation: Theory and Applications. Philadelphia, PA: SIAM.

[22] Isidori, A. (1995). Nonlinear Control Systems (3rd ed.). London: Springer-Verlag.

[23] Isidori, A. (1997). A remark on the problem of semiglobal nonlinear output regulation. IEEE Trans. Automatic Control, 42, 1734-1738. 
[24] Isidori, A., \& Byrnes, C.I. (1990). Output regulation of nonlinear systems. IEEE Trans. Automatic Control, 35, 131-140.

[25] Isidori, A., \& Marconi, L. (2012). Shifting the internal model from control input to controlled output in nonlinear output regulation. Proc. of $51^{\text {st }}$ IEEE Conf. Decision and Control, Maui, USA (pp. 4900-4905).

[26] Jayawardhana, B., \& Weiss, G. (2008). Tracking and disturbance rejection for fully actuated mechanical systems. Automatica, 44, 2863-2868.

[27] Jayawardhana, B., \& Weiss, G. (2009). State convergence of passive nonlinear systems with an $L^{2}$ input. IEEE Trans. Automatic Control, 54, 1723-1727.

[28] Kassakian, J.G., Schlecht, M.F., \& Verghese, G.C. (1991). Principles of Power Electronics. Reading, MA: Addison-Wesley.

[29] Khalil, H.K. (1994). Robust servomechanism output feedback controllers for feedback linearizable systems. Automatica, 30, 1587-1599.

[30] Khalil, H.K. (2002). Nonlinear Systems. New Jersey: Prentice Hall.

[31] Knobloch, H.W., Isidori, A., \& Flockerzi, D. (1993). Topics in Control Theory. Basel: Birkhäuser-Verlag.

[32] Krein, P.T., Bentsman, J., Bass, R.M., \& Lesieutre, B.C. (1990). On the use of averaging for the analysis of power electronic systems. IEEE Trans. Power Electronics, 5, 182-190.

[33] Margaliot, M., \& Weiss, G. (2010). The low-frequency distortion in D-class amplifiers. IEEE Trans. Circuits and Systems II, 57, 772-776.

[34] Marsden, J.E. (1974). Elementary Classical Analysis. San Francisco: W.H. Freeman and Company.

[35] Naim, R., Weiss, G., \& Ben-Yaakov, S. (1997). $H^{\infty}$ control applied to boost power converters. IEEE Trans. Power Electronics, 12, 677-683.

[36] Natarajan, V., \& Weiss, G. (2013). Behavior of a stable nonlinear infinite-dimensional system under the influence of a nonlinear exosystem. Proc. of the $1^{\text {st }}$ IFAC Workshop on Control of Systems Modeled by PDEs, Paris (pp. 155-160).

[37] Natarajan, V., \& Weiss, G. (2014). Minimal order controllers for output regulation of locally stable nonlinear systems. 53rd IEEE Conf. on Decision and Control, Los Angeles (pp. 4709-4714).

[38] Ping, Z., \& Huang, J. (2011). Global robust output regulation for a class of multivariable systems and its application to a motor drive system. Proc. of American Control Conference, San Francisco, USA (pp. 4560-4565).

[39] Priscoli, F.D. (1993). Robust tracking for a class of nonlinear plants achieved via a linear controller. Proc. of 32 $2^{\text {nd }}$ IEEE Conf. Decision and Control, San Antonio, USA (pp. 3550-3555).

[40] Priscoli, F.D. (1997). Sufficient conditions for robust tracking in nonlinear systems. Int. J. Control, 67, 825-836. 
[41] Rebarber, R., \& Weiss, G. (2003). Internal model based tracking and disturbance rejection for stable well-posed systems. Automatica, 39, 1555-1569.

[42] Rehak, B. (2012). Alternative method of solution of the regulator equation: $L^{2}$-space approach. Asian Journal of Control, 14, 1150-1154.

[43] Rehak, B., \& Celikovsky, S. (2008). Numerical method for the solution of the regulator equation with application to nonlinear tracking. Automatica, 44, 1358-1365.

[44] Scherer, C., Gahinet, P. \& Chilali, M. (1997). Multiobjective output-feedback control via LMI optimization. IEEE. Trans. Automatic Control, 42, 896-911.

[45] Serrani, A., \& Isidori, A. (2000). Global robust output regulation for a class of nonlinear systems. Systems \& Control Letters, 39, 133-139.

[46] Serrani, A., Isidori, A., \& Marconi, L. (2000). Semiglobal robust output regulation of minimum-phase nonlinear systems. Int. J. Robust Nonlinear Control, 10, 379-396.

[47] Syrmos, V.L., Abdallah, C.T., Dorato, P., \& Grigoriadis, K. (1997). Static output feedback - A survey. Automatica, 33, 125-137.

[48] Weiss, G., \& Natarajan, V. (2014). Tracking controller for output voltage regulation in a boost converter. IEEE Conv. of Electr. \& Electronics Eng. in Israel, Eilat.

[49] Xi, Z., \& Ding, Z. (2007). Global adaptive output regulation of a class of nonlinear systems with nonlinear exosystems. Automatica, 43, 143-149. 